\title{
Homotopical decompositions of simplicial and Vietoris Rips complexes
}

\author{
Wojciech Chachólski ${ }^{1}$ (D) Alvin Jin ${ }^{1} \cdot$ Martina Scolamiero ${ }^{1}$. \\ Francesca Tombari ${ }^{1}$
}

Received: 11 February 2020 / Accepted: 19 February 2021 / Published online: 3 May 2021

(c) The Author(s) 2021

\begin{abstract}
Motivated by applications in Topological Data Analysis, we consider decompositions of a simplicial complex induced by a cover of its vertices. We study how the homotopy type of such decompositions approximates the homotopy of the simplicial complex itself. The difference between the simplicial complex and such an approximation is quantitatively measured by means of the so called obstruction complexes. Our general machinery is then specialized to clique complexes, Vietoris-Rips complexes and Vietoris-Rips complexes of metric gluings.
\end{abstract}

Keywords Vietoris-Rips complexes · Metric gluings $\cdot$ Closed classes $\cdot$ Homotopy push-outs

Mathematics Subject Classification $55 \cdot 55 \mathrm{~N} 31 \cdot 62 \mathrm{R} 40$

\section{Introduction}

Homology is an example of an invariant that is both calculable and geometrically informative. These two features are key reasons why invariants derived from homology are fundamental in Algebraic Topology in general and in Topological Data Analysis (TDA), see (Carlsson 2009), in particular. The most effective strategies for calculating

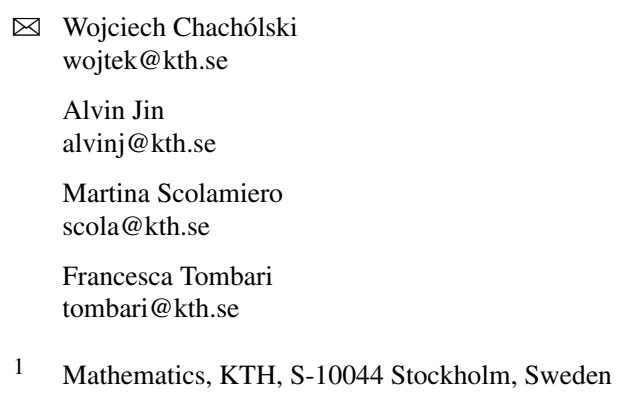


homology in algebraic topology are based on the fact that homology converts homotopy push-outs of spaces into Mayer-Vietoris exact sequences. Thus decomposing a space into a homotopy push-out enables the extraction of homologies of the decomposed space (global information) from the homologies of the spaces in the push-out (local information).

The ability to extract global information from local is important. What is meant by local information however depends on the input and the description of considered spaces. For example what is often understood as local information in TDA differs from the local information described above (push-out decomposition).

In TDA the input is typically a finite pseudo-metric space. This information is then converted into spacial information and in this article we focus on the so called Vietoris-Rips construction (Hausmann 1995) for that purpose. Homologies extracted from this space give rise to invariants of the metric space used in TDA such as persistent homology (Cagliari et al. 2001; Delfinado and Edelsbrunner 1995; Edelsbrunner and Harer 2008; Ferri 1995; Frosini and Landi 1999), barcodes (Carlsson et al. 2005), stable ranks (Chachólski and Riihimäki 2020; Scolamiero et al. 2017), or persistent landscapes (Bubenik 2015). This conversion process, from metric into spacial information, does not in general transform the gluing of metric spaces (Taubes 1996) into homotopy push-outs and homotopy colimits of simplicial complexes. The aim of this paper is to understand how close such metric space decompositions are to decompositions into homotopy push-outs. Our work was inspired by Adamaszek et al. (2018) and Adamaszek et al. (2020), and grew out from realising that analogous statements hold true for arbitrary simplicial complexes and not just Vietoris-Rips complexes. To get these general statements we use categorical techniques. This enables us to prove stronger results using arguments that for us are more transparent.

The most general input for our investigation is a simplicial complex $K$ and a cover $X \cup Y=K_{0}$ of its set of vertices. In this article we study the map $K_{X} \cup K_{Y} \subset K$ where $K_{X}$ and $K_{Y}$ are subcomplexes of $K$ consisting of all these simplices of $K$ which are subsets of $X$ and $Y$ respectively. The goal is to estimate the homotopy fibers of this inclusion. We do that in terms of obstruction complexes $\operatorname{St}(\sigma, X \cap Y):=\{\mu \subset$ $X \cap Y|0<| \mu \mid$ and $\mu \cup \sigma \in K$ \} indexed by simplices $\sigma$ in $K$ (see Definition 5.1). Our main result, Theorem 8.6, states that the homotopy fibers of $K_{X} \cup K_{Y} \subset K$ are in the same closed class (see Paragraph 3.5, and Chachólski (1996), Dror Farjoun (1995), Farjoun (1996)) as the obstruction complexes $\operatorname{St}(\sigma, X \cap Y)$ for all $\sigma$ in $K$ such that $\sigma \cap X \neq \emptyset, \sigma \cap Y \neq \emptyset$, and $\sigma \cap X \cap Y=\emptyset$. For instance (see Corollary 8.7.1) if, for all such $\sigma$, the obstruction complex $\operatorname{St}(\sigma, X \cap Y)$ is contractible, then $K_{X} \cup K_{Y} \subset$ $K$ is a weak equivalence and consequently $K$ decomposes as a homotopy push-out hocolim $\left(K_{X} \hookleftarrow K_{X \cap Y} \hookrightarrow K_{Y}\right)$ leading to a Mayer-Vietoris exact sequence. Another instance of our result (see Corollary 8.7.2) states that if these obstruction complexes have trivial homology in degrees not exceeding $n$, then so do the homotopy fibers of $K_{X} \cup K_{Y} \subset K$ and consequently this map induces an isomorphism on homology in degrees not exceeding $n$, leading to a partial Mayer-Vietoris exact sequence. Yet another consequence (see Corollary 8.7.3) is that if these obstruction complexes have $p$-torsion homology for a prime $p$, then, for any field $F$ of characteristic different than $p$, the inclusion $K_{X} \cup K_{Y} \subset K$ induces an isomorphism on homology with 
coefficients in $F$ leading again to a Mayer-Vietoris exact sequence (see (Adamaszek 2014) for examples of relevant complexes where torsion is present).

An essential assumption to guarantee stability of the formation of Vietoris-Rips complexes is the triangular inequality of the input distance spaces. We thus believe it is important to investigate and understand the role of the triangular inequality in persistent homology. That is why in Sect. 10 we specialise our theorem about the cellularity of the homotopy fibers of the inclusion $K_{X} \cup K_{Y} \subset K$ to the case when $K$ is clique and give some conditions that imply the assumptions of the theorem in this case. Obtained results generalize the statements proven in Adamaszek et al. (2018) and Adamaszek et al. (2020) for Vietoris-Rips complexes. We would like to stress that our results are not just for Vietoris-Rips complexes but hold more generally for clique complexes. In particular, the triangular inequality of the input distance space is not needed for these statements to hold. However in order to prove Theorem 12.5 it is essential that the considered complex is the Vietoris-Rips complex of the metric gluing of pseudo-metric spaces for which the triangular inequality is satisfied. This theorem gives 2-connectedness of the relevant homotopy fibers and hence can be used to calculate $H_{1}$ and $H_{0}$ of the gluing in terms of $H_{1}$ 's and $H_{0}$ 's of the components and the intersection.

Putting the results of Adamaszek et al. (2018) and Adamaszek et al. (2020) in a broader context was a mathematical motivation for this article. Presenting mathematical foundations for a strategy of calculating persistent homology based on decompositions was another motivation. Even in the case a metric is obtained by gluing together several metrics defined locally (metric gluing), homology of its Vietoris-Rips complex is a global invariant. That is one of the reason why there are no efficient parallelisable algorithms for calculating persistent homology in general. That prohibits calculating persistent homology based invariants for metrics constructed out of large networks such as large-scale collections of GPS traces for vehicles and pedestrians or track motions of high-energy particles moving along filamentary trajectories (see Aanjaneya et al. 2011). However since strategies based on decompositions would allow parallelisation, they can be more useful for such large metrics. For example one can use Theorem 12.5 to calculate the 1-st persistent homology of certain metrics in terms of local information. More generally one can use Theorem 8.6 to estimate the difference between the global persistent homology of a distance and the persistent homologies of its local pieces appropriately glued. Theorem 8.6 not only can be used to estimate homological differences between global and local persistent homologies but also can be used to estimate the difference on the space level. Illustrating this is the reason we wrote Sect. 13.

Following the mathematical foundations presented in this article, next steps in developing more effective strategies of calculating persistent homology using decompositions would require understanding effects of decompositions into homotopy colimits of diagrams indexed by more complex categories. Categories of interest are higher dimensional cubes (corresponding to covers into more than two sets), or zigzags (related to the formation of Reeb graphs and the mapper algorithms) or categories related to groups and group actions. 


\section{Homotopy primer on simplicial sets}

We refer the reader to Curtis (1967), Goerss and Jardine (1999) for an overview of how to do homotopy theory on simplicial sets. To make the paper more self contained however, we recall in this section basic homotopical constructions which play a fundamental role in our statements. We consider the standard model structure on the category of simplicial sets where weak equivalences are the maps inducing bijections on all the homotopy groups with respect to any choice of a base point.

A model structure guarantees that an arbitrary map $f: X \rightarrow Y$ of simplicial sets can be factored in two ways, a weak equivalence followed by a fibration and a cofibration followed by a weak equivalence:

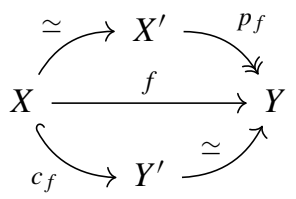

Once such factorisations of $f$ are chosen, we refer to the fiber $p_{f}^{-1}(y)$ (of the fibration $p_{f}$ ) as a homotopy fiber of $f$ over $y$, and to the quotient $Y^{\prime} / c_{f}(X)$ as a homotopy cofiber of $f$. The homotopy types of homotopy fibers and of $f$ do not depend on the choice of the factorisations and we use the symbols $\operatorname{Fib}(f, y)$ and $\operatorname{Cof}(f)$ to denote them. Homotopy fibers and cofibers of $f$ fit into the following exact sequences for every natural number $k$, where $H_{\bullet}$ is a homology functor (possibly extraordinary):

$$
\begin{aligned}
& \pi_{k+1}(X, x) \stackrel{\pi_{k+1}(f)}{\longrightarrow} \pi_{k+1}(Y, f(x)) \downarrow \\
& \pi_{k}(\operatorname{Fib}(f, f(x))) \longrightarrow \pi_{k}(X, x) \stackrel{\pi_{k}(f)}{\longrightarrow} \pi_{k}(Y, f(x)) \\
& H_{k+1}(Y) \longrightarrow H_{k+1}(\operatorname{Cof}(f)) \\
& \downarrow \\
& H_{k}(X) \stackrel{H_{k}(f)}{\longrightarrow} H_{k}(Y) \longrightarrow H_{k}(\operatorname{Cof}(f))
\end{aligned}
$$

In particular, $\operatorname{Fib}(f, y)$ is $n$-connected for every $y$, if and only if, for every $x$ in $X$, $\pi_{k}(f)$ is a bijection for all $k \leq n$ and a surjection for $k=n+1$. Thus the homotopy fibers measure to what extent $f$ is a weak equivalence.

Homotopy fibers and cofibers can be assigned not only to 1-dimensional cubes, i.e., maps, but also to higher dimensional cubes. Here we just illustrate how to do that for a commutative square i.e., a 2-dimensional cube. Let us consider a commutative square represented by the diagonal $A B C D$ in the following diagram. We can use the same factorisation axiom of the model structure to fit this square into the following commutative 3-dimensional cube where the indicated arrows are respectively weak equivalences, fibrations, cofibrations, the square $A B^{\prime} C Q$ is a push-out, and the square $P B C^{\prime} D$ is a pull-back: 


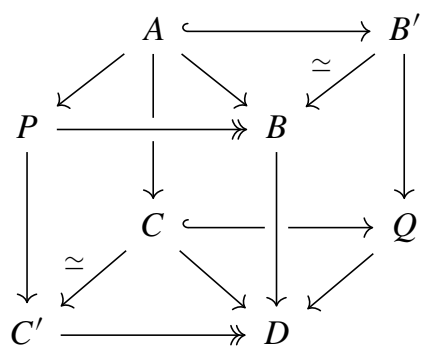

The maps $A \rightarrow P$ and $Q \rightarrow D$ are then uniquely determined by the commutativity of this diagram and the fact that the squares $P B C^{\prime} D$ and $A B^{\prime} C Q$ are pull-back and pushout respectively. If the map $Q \rightarrow D$ is a weak equivalence, then the square $A B C D$ is called a homotopy push-out. If $A \rightarrow P$ is a weak equivalence, then $A B C D$ is called a homotopy pull-back. For example, a push-out square $A B C D$ for which $A \rightarrow B$ is a cofibration is a homotopy push-out, and a pull-back square $A B C D$ for which $C \rightarrow D$ is a fibration is a homotopy pull-back.

Consider now two composable maps $X-f \rightarrow Y-g \rightarrow Z$. These maps are said to form a cofibration sequence, respectiely a fibration sequence, if they can be fitted into a commutative square:

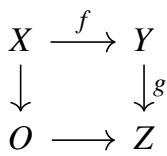

where $O$ is contractible and the square is homotopy push-out, respectively homotopy pull-back. For example if $g: Y \rightarrow Z$ is a fibration, then $g^{-1}(z) \longleftrightarrow Y-g \rightarrow Z$ form a fibration sequence. If $f: X \rightarrow Y$ is a cofibration then $f$ together with the quotient map $Y \rightarrow Y / f(X)$ is a cofibration sequence.

If $X-f \rightarrow Y-g \rightarrow Z$ is a fibration sequence, then $X$ is a homotopy fiber of $g: Y \rightarrow Z$ and, for every natural number $k$, there is an exact sequence:

$$
\begin{aligned}
\pi_{k+1}(Y, f(x)) \stackrel{\pi_{k+1}(g)}{\longrightarrow} \pi_{k+1}(Z, g(f(x))) & \\
\downarrow & \stackrel{\downarrow}{\pi_{k}(X, x)} \stackrel{\pi_{k}(f)}{\longrightarrow} \pi_{k}(Y, f(x)) \stackrel{\pi_{k}(g)}{\longrightarrow} \pi_{k}(Z, g(f(x)))
\end{aligned}
$$

If $X-f \rightarrow Y-g \rightarrow Z$ is a cofibration sequence, then $Z$ is the homotopy cofiber of $f: X \rightarrow Y$ and, for every natural number $k$, there is an exact sequence, where $H_{\bullet}$ is a homology functor: 


$$
\begin{aligned}
& H_{k+1}(Y) \stackrel{H_{k+1}(g)}{\longrightarrow} H_{k+1}(Z) \\
& \downarrow \\
& H_{k}(X) \stackrel{H_{k}(f)}{\longrightarrow} H_{k}(Y) \stackrel{H_{k}(g)}{\longrightarrow} H_{k}(Z)
\end{aligned}
$$

\section{Small categories and simplicial sets}

In this section we recall some elements of a convenient language for describing and discussing homotopical properties of small categories. The key role here is played by the nerve construction (Latch et al. 1979, [25]) that transforms small categories into simplicial sets.

Here is a list of definitions and characterizations of various homotopical notions for small categories and some statements regarding these notions.

3.1 Let $\mathcal{C}$ be a property of simplicial sets, such as being contractible, $n$-connected, having $p$-torsion integral reduced homology, or having trivial reduced homology in some degrees. By definition a small category $I$ satisfies $\mathcal{C}$ if and only if its nerve $N(I)$ satisfies $\mathcal{C}$.

3.2 Let $\mathcal{C}$ be a property of maps of simplicial sets, such as being a weak equivalence, a homology isomorphism, or having $n$-connected homotopy fibers. By definition, a functor $f: I \rightarrow J$ between small categories satisfies $\mathcal{C}$ if and only if the map of simplicial sets $N(f): N(I) \rightarrow N(J)$ satisfies $\mathcal{C}$.

3.3 Functors $f, g: I \rightarrow J$ are homotopic if the maps $N(f), N(g): N(I) \rightarrow N(J)$ are homotopic. For example, if there is a natural transformation $\phi: f \Rightarrow g$ between $f$ and $g$, then $f$ and $g$ are homotopic.

Assume $I$ has a terminal object $t$. Then there is a unique natural transformation from the identity functor id: $I \rightarrow I$ to the constant functor $t: I \rightarrow I$ with value $t$. The identity functor is therefore homotopic to the constant functor, and consequently $I$ is contractible. By a similar argument, a category with an initial object is also contractible.

3.4 A commutative square of small categories is called a homotopy push-out (pullback) if after applying the nerve construction the obtained commutative square of simplicial sets is a homotopy push-out (pull-back).

3.5 Recall that a collection $\mathcal{C}$ of simplicial sets is closed if it contains a nonempty simplicial set and it is closed under weak equivalences and homotopy colimits indexed by arbitrary small contractible categories (Chachólski 1996, Corollary 7.7). Any closed collection contains all contractible simplicial sets (Chachólski 1996, Proposition 4.5). If a closed collection contains an empty simplicial set, then it contains all simplicial sets.

The following are some examples of collections of simplicial sets that are closed: contractible simplicial sets, $n$-connected simplicial sets, connected simplicial sets having $p$-torsion reduced integral homology, simplicial sets having trivial reduced 
homology with some fixed coefficients up to a given degree, and simplicial sets which are acyclic with respect to some (possibly not ordinary) homology theory.

Let $\mathcal{C}$ be a closed collection of simplicial sets and $f: I \rightarrow J$ be a functor between small categories. We say that homotopy fibers of $f$ satisfy $\mathcal{C}$ if the homotopy fibers of $N(f): N(I) \rightarrow N(J)$, over any component in $N(J)$, belong to $\mathcal{C}$.

3.6 Let $f: I \rightarrow J$ be a functor between small categories. For an object $j$ in $J$, the symbol $j \uparrow f$ denotes the category whose objects are pairs $(i, \alpha: j \rightarrow f(i))$ consisting of an object $i$ in $I$ and a morphism $\alpha: j \rightarrow f(i)$ in $J$. The set of morphisms in $j \uparrow f$ between $(i, \alpha: j \rightarrow f(i))$ and $\left(i^{\prime}, \alpha^{\prime}: j \rightarrow f\left(i^{\prime}\right)\right)$ is by definition the set of morphisms $\beta: i \rightarrow i^{\prime}$ in $I$ for which the following triangle commutes:

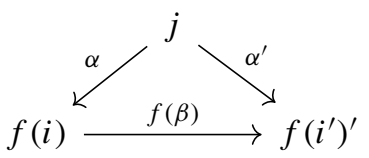

The composition in $j \uparrow f$ is given by the composition in $I$.

For an object $j$ in $J$, the symbol $f \downarrow j$ denotes the category whose objects are pairs $(i, \alpha: f(i) \rightarrow j)$ consisting of an object $i$ in $I$ and a morphism $\alpha: f(i) \rightarrow j$ in $J$. The set of morphisms in $f \downarrow j$ between $(i, \alpha: f(i) \rightarrow j)$ and $\left(i^{\prime}, \alpha^{\prime}: f\left(i^{\prime}\right) \rightarrow j\right)$ is by definition the set of morphisms $\beta: i \rightarrow i^{\prime}$ in $I$ for which the following triangle commutes:

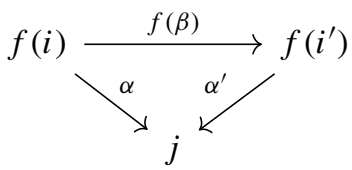

The composition in $f \downarrow j$ is given by the composition in $I$.

Theorem 3.7 (Chachólski 1996, Theorem 9.1) Let C be a closed collection of simplicial sets and $f: I \rightarrow J$ be a functor between small categories.

1. If, for every $j, f \downarrow j$ satisfies $\mathcal{C}$, then so do the homotopy fibers of $f$.

2. If, for every $j, j \uparrow f$ satisfies $\mathcal{C}$, then so do the homotopy fibers of $f$.

Depending on the choice of a closed collection, Theorem 3.7 leads to:

Corollary 3.8 Let $f: I \rightarrow J$ be a functor between small categories.

1. If, for every $j, f \downarrow j$ (respectively $j \uparrow f$ ) is contractible, then $f$ is a weak equivalence.

2. If, for every $j, f \downarrow j$ (respectively $j \uparrow f$ ) is $n$-connected for some $n \geq 0$, then the homotopy fibers of $f$ are $n$-connected. Thus in this case $f$ induces an isomorphism on homotopy groups in degrees $0, \ldots, n$ and a surjection in degree $n+1$.

3. If, for every $j, f \downarrow j$ (respectively $j \uparrow f$ ) is connected and has $p$-torsion reduced integral homology in degrees not exceeding $n(n \geq 0)$, then the homotopy fibers of $f$ are connected and have p-torsion reduced integral homology in degrees not 
exceeding $n$. Thus in this case, for primes $q \neq p, f$ induces an isomorphism on $H_{*}(-, \mathbf{Z} / q)$ for $* \leq n$ and a surjection on $H_{n+1}(-, \mathbf{Z} / q)$.

4. If, for every $j, f \downarrow j$ (respectively $j \uparrow f$ ) is acyclic with respect to some homology theory, then $f$ induces an isomorphism in this homology theory.

\section{Simplicial complexes and small categories}

4.1 Fix a set $\mathcal{U}$ called a universe. A simplicial complex is a collection $K$ of finite nonempty subsets of $\mathcal{U}$ that satisfies the following requirement: if $\sigma \subset \mathcal{U}$ is in $K$, then every non-empty subset of $\sigma$ is also in $K$.

Let $X \subset \mathcal{U}$ be a subset. The collection $\{\{x\} \mid x \in X\}$, consisting of singletons in $X$, is a simplicial complex denoted also by $X$, called the discrete simplicial complex on $X$. The collection $\{\sigma \subset X|1 \leq| \sigma \mid<\infty\}$ of all finite nonempty subsets of $X$ is also a simplicial complex denoted by $\Delta[X]$ and called the simplex on $X$. A simplicial complex is called a standard simplex if it is of the form $\Delta[X]$ for some $X \subset \mathcal{U}$. The simplex $\Delta[\emptyset]$ is called the empty simplex or the empty simplicial complex.

4.2 Let $K$ be a simplicial complex. An element $\sigma$ in $K$ is called a simplex of $K$ of dimension $|\sigma|-1$. The set of $n$-dimensional simplices in $K$ is denoted by $K_{n}$. An element $x \in \mathcal{U}$ is called a vertex of $K$ if $\{x\}$ is a simplex in $K$. The assignment $x \mapsto\{x\}$ is a bijection between the set of vertices in $K$ and the set of its 0 -dimensional simplices $K_{0}$. We use this bijection to identify these sets. Thus we are going to refer to 0 -dimensional simplices in $K$ also as vertices.

4.3 If $\left\{K^{i}\right\}_{i \in I}$ is a family of simplicial complexes, then both the intersection $\cap_{i \in I} K^{i}$ and the union $\cup_{i \in I} K^{i}$ are also simplicial complexes. In particular, if $K$ is a simplicial complex and $X \subset \mathcal{U}$ is a subset, then the intersection $K \cap \Delta[X]$ is a simplicial complex consisting of the elements of $K$ that are subsets of $X$. This intersection is called the restriction of $K$ to $X$ and is denoted by $K_{X}$.

Note that $\Delta[X] \cap \Delta[Y]=\Delta[X \cap Y]$. Thus the intersection of standard simplices (see 4.1) is again a standard simplex, which can possibly be empty.

Let $L$ and $K$ be simplicial complexes. If $L \subset K$, then $L$ is called a subcomplex of $K$. Being a subcomplex is a partial order relation on the collection of all simplicial complexes which gives this collection the structure of a lattice. The union is the join and the intersection is the meet.

The collection $\cup_{0 \leq i \leq n} K_{i}$ is a subcomplex of $K$ called the $n$-th skeleton of $K$ and denoted by $\mathrm{sk}_{n} K$.

4.4 A map between two simplicial complexes $K$ and $L$ is by definition a function $\phi: K \rightarrow L$ for which there exists a function $f: K_{0} \rightarrow L_{0}$ such that $\phi(\sigma)=$ $\cup_{x \in \sigma} f(\{x\})$ for all $\sigma$ in $K$. In particular $\phi(\{x\})=f(\{x\})$ for every vertex $x$ in $K$. Thus $f$ is uniquely determined by $\phi$ and we often use the symbol $\phi_{0}$ to denote $f$. If $K$ and $L$ are fixed, then $\phi$ is determined by $f=\phi_{0}$. The inclusion $L \subset K$ of a subcomplex is an example of a map.

For any simplicial complex $K$, the inclusions $K_{0} \subset K \subset \Delta\left[K_{0}\right]$, between the discrete simplicial complex $K_{0}, K$, and the simplex $\Delta\left[K_{0}\right]$ on $K_{0}$ are maps of simplicial 
complexes. The induced functions on the set of vertices for these two inclusions are given by the identity function id: $K_{0} \rightarrow K_{0}$.

4.5 Classically, the geometrical realization is used to define and study homotopical properties of simplicial complexes. For example, a commutative square of simplicial complexes is called a homotopy push-out (pull-back) if after applying the realization, the obtained commutative square of spaces is a homotopy push-out (pull-back). For instance two simplicial complexes $K$ and $L$ fit into the following commutative diagram of subcomplex inclusions:

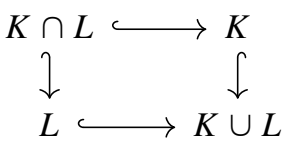

By applying the realization construction to this square, we obtain a commutative square of spaces which is a push-out and hence a homotopy push-out as the maps involved are cofibrations.

There are situations however when another way of extracting homotopical properties of simplicial complexes is more convenient. In the rest of this section, we recall how one can retrieve and study such information by first transforming simplicial complexes into small categories and then using the nerve construction as explained in Sect. 3.

4.6 Let $K$ be a simplicial complex. The simplex category of $K$, denoted also by the same symbol $K$, is by definition the inclusion poset of its simplices. Thus, the objects of $K$ are the simplices in $K$ and the sets of morphisms are either empty or contain only one element:

$$
\operatorname{mor}_{K}(\sigma, \tau) \mid= \begin{cases}1 & \text { if } \sigma \subset \tau \\ 0 & \text { otherwise }\end{cases}
$$

If $\phi: K \rightarrow L$ is a map of simplicial complexes, then the assignment $\sigma \mapsto \phi(\sigma)$ is a functor of simplex categories. We denote this functor also by the symbol $\phi: K \rightarrow L$. Not all functors between $K$ and $L$ are of such a form.

The geometrical realization of a simplicial complex is weakly equivalent to the realization of the nerve of this simplicial complex. Thus to describe homotopical properties of simplicial complexes we can either use their geometrical realizations or the nerves of their simplex categories.

Proposition 4.7 Let $K$ be a simplicial complex, $n \geq 0$ a natural number, and $P \subset K a$ subposet such that $k_{n+1} K \subset P$. Then the homotopy fibers of $P \subset K$ are $n$-connected. In particular the functor $P \subset K$ induces an isomorphism on homotopy and integral homology groups in degrees $0, \ldots, n$ and a surjection in degree $n+1$.

Proof Let us denote the inclusion $\mathrm{sk}_{n+1} K \subset P$ functor by $f$. For every simplex $\sigma$ in $P$, the category $f \downarrow \sigma$ is the simplex category of $\operatorname{sk}_{n+1} \Delta[\sigma]$. Since $\operatorname{sk}_{n+1} \Delta[\sigma]$ is $n$-connected, Corollary 3.8.2 implies that the homotopy fibers of $f$ are $n$-connected. 
The same argument gives $n$-connectedness of the homotopy fibers of the inclusion sk $_{n+1} K \subset K$. Consequently the homotopy fibers of the inclusion $P \subset K$ are also $n$-connected.

4.8 Let $K$ be a simplicial complex. Define the star of a simplex $\sigma$ in $K$ to be $\operatorname{St}(\sigma):=$ $\{\mu \in K \mid \sigma \cup \mu \in K\}$. Note that $\operatorname{St}(\sigma)$ is a subcomplex of $K$ which contains $\Delta[\sigma]$. If $\tau \subset \sigma$, then $\operatorname{St}(\sigma) \subset \operatorname{St}(\tau)$, and thus $\sigma \mapsto \operatorname{St}(\sigma)$ is a contravariant functor indexed by the simplex category $K$ with values in the inclusion poset of subcomplexes of $K$.

The star of any simplex is contractible. More generally:

Proposition 4.9 Let $\sigma$ be a simplex in $K$. Then, for any proper subset $S \subsetneq \sigma$, the collection $L:=\{\mu \in K \mid \mu \cap S=\emptyset$ and $\sigma \cup \mu \in K\}$ is a contractible simplicial complex (note that if $S=\emptyset$, then $L=S t(\sigma)$ ).

Proof For all $\mu$ in $L$, the inclusions $\mu \hookrightarrow \mu \cup(\sigma \backslash S) \hookleftarrow \sigma \backslash S$ form natural transformations between:

- the identity functor id: $L \rightarrow L, \mu \mapsto \mu$,

- $L \rightarrow L$, given by $\mu \mapsto \mu \cup(\sigma \backslash S)$,

- and the constant functor $L \rightarrow L, \mu \mapsto \sigma \backslash S$.

The identity functor id: $L \rightarrow L$ is therefore homotopic to the constant functor and consequently $L$ is contractible.

4.10 Let $K$ be a simplicial complex. It's simplex $\tau$ is called central if $K=\operatorname{St}(\tau)$, i.e., if for any simplex $\sigma$ in $K$, the set $\sigma \cup \tau$ is also a simplex in $K$. For example, if $X \subset \mathcal{U}$ is non empty, then all simplices in $\Delta[X]$ (see 4.1) are central. If $\tau$ is a central simplex in $K$, then so is any subset $\tau^{\prime} \subset \tau$. According to Proposition 4.9, a simplicial complex that has a central simplex is contractible.

4.11 Let $K$ and $L$ be simplicial complexes. If $K \cap L=\emptyset$, then we define their join $K * L$ to be the simplicial complex consisting of all subsets of $\mathcal{U}$ of the form $\sigma \cup \tau$ where $\sigma$ is in $K$ and $\tau$ is in $L$. The join is only defined for disjoint simplicial complexes. The set of vertices $(K * L)_{0}$ is given by the (disjoint) union $K_{0} \cup L_{0}$. Note that $K * \Delta[\emptyset]=K$. If $\sigma \in L$ is central in $L$, then it is central in $K * L$. Thus, for any non-empty subset $X \subset \mathcal{U} \backslash K_{0}$, the join $K * \Delta[X]$ is contractible. Furthermore the join commutes with unions and intersections: if $\left(K_{1} \cup K_{2}\right) \cap L=\emptyset$, then $\left(K_{1} \cup K_{2}\right) * L=\left(K_{1} * L\right) \cup\left(K_{2} * L\right)$ and $\left(K_{1} \cap K_{2}\right) * L=\left(K_{1} * L\right) \cap\left(K_{2} * L\right)$. This can be used to show that, for any choice of a base-points in $K$ and $L$, the join $K * L$ has the homotopy type of the suspension of the smash $\Sigma(|K| \wedge \mid L])$. In particular if $K$ is $n$-connected and $L$ is $m$-connected, then $K * L$ is $n+m+1$-connected.

\section{One outside point}

In this section we recall how the homotopy type of a simplical complex changes when a vertex is added. We start with defining subcomplexes that play an important role in describing such changes. These complexes are essentially used throughout the entire paper. 
Fig. 1 The simplicial complex $K$ has five vertices in total. The set $A=K_{0} \backslash\left\{v_{0}, v_{1}\right\}$ here consists of three points $\left\{a_{0}, a_{1}, a_{2}\right\}$. The obstruction complex associated to the simplex $\sigma, \operatorname{St}(\sigma, A)$, contains only vertices $a_{0}$ and $a_{1}$, but not the edge $\left\{a_{0}, a_{1}\right\}$

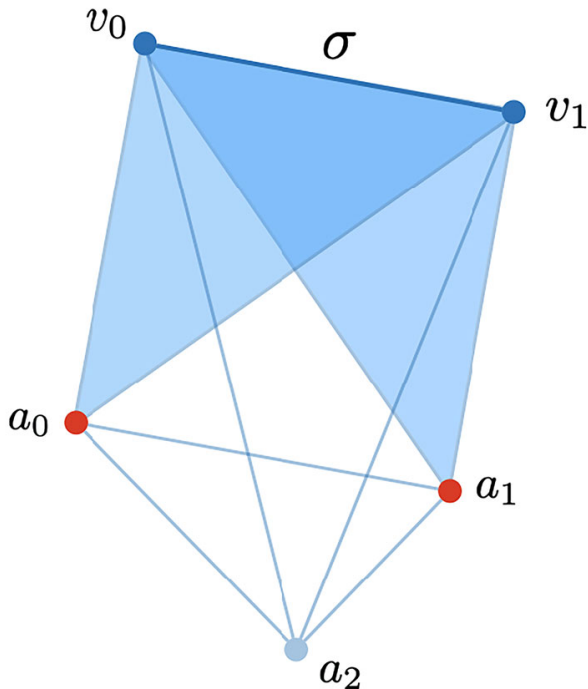

Definition 5.1 Let $K$ be a simplicial complex and $A \subset \mathcal{U}$ be a subset. For a simplex $\sigma$ in $K$, define the obstruction complex:

$$
\operatorname{St}(\sigma, A):=\{\mu \subset A|0<| \mu \mid \text { and } \mu \cup \sigma \in K\}=K_{A} \cap \operatorname{St}(\sigma)
$$

If $\mu$ belongs to $\operatorname{St}(\sigma, A)$, then so does any of its non empty finite subsets. Thus $\operatorname{St}(\sigma, A)$ is a simplicial complex. It is a subcomplex of $K_{A}$. Note that the complex $\operatorname{St}(\sigma, A)$ may be empty. If $\tau \subset \sigma$, then $\operatorname{St}(\sigma, A) \subset \operatorname{St}(\tau, A)$ and hence $\sigma \mapsto \operatorname{St}(\sigma, A)$ is a contravariant functor indexed by the simplex category $K$ with values in the inclusion poset of subcomplexes of $K_{A}$ (see 4.8). Figure 1 illustrates an example of an obstruction complex.

Fix a vertex $v$ in $K$. Any simplex in $K$ either contains $v$ or it does not. This means $K=K_{K_{0} \backslash\{v\}} \cup \operatorname{St}(v)$ and hence we have a (homotopy) push-out square:

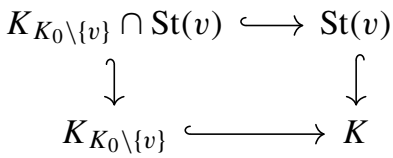

By definition $K_{K_{0} \backslash\{v\}} \cap \operatorname{St}(v)=\operatorname{St}\left(v, K_{0} \backslash\{v\}\right)$, which coincides with what is also called the link of a vertex $v$ (see Maunder 1996). Proposition 4.9 gives contractibility of $\operatorname{St}(v)$. The simplicial complex $K$ fits therefore into the following homotopy cofiber sequence:

$$
\operatorname{St}\left(v, K_{0} \backslash\{v\}\right) \hookrightarrow K_{K_{0} \backslash\{v\}} \hookrightarrow K
$$

Here are some basic consequences of this fact: 
Corollary 5.2 Let $v$ be a vertex in a simplical complex $K$.

1. If $\operatorname{St}\left(v, K_{0} \backslash\{v\}\right)$ is contractible, then $K_{K_{0} \backslash\{v\}} \subset K$ is a weak equivalence.

2. If $S t\left(v, K_{0} \backslash\{v\}\right)$ is n-connected for a natural number $n \geq 0$, then the map $K_{K_{0} \backslash\{v\}} \subset K$ induces an isomorphism on homotopy groups in degrees $0, \ldots, n$ and a surjection in degree $n+1$.

3. If $\operatorname{St}\left(v, K_{0} \backslash\{v\}\right)$ is connected and has p-torsion reduced integral homology in degrees not exceeding $n(n \geq 0)$, then for a prime $q$ not dividing $p, K_{K_{0} \backslash\{v\} \subset} \subset$ $K$ induces an isomorphism on $H_{*}(-, \mathbf{Z} / q)$ for $* \leq n$ and a surjection on $H_{n+1}(-, \mathbf{Z} / q)$.

4. If St $\left(v, K_{0} \backslash\{v\}\right)$ is acyclic with respect to some homology theory, then $K_{K_{0} \backslash\{v\}} \subset K$ is this homology isomorphism.

\section{Two outside points}

Let us fix two distinct vertices $v_{0}$ and $v_{1}$ in a simplicial complex $K$. Note that $\left(K_{0} \backslash\left\{v_{0}\right\}\right) \cup\left(K_{0} \backslash\left\{v_{1}\right\}\right)=K_{0}$. In this section we are going to investigate the inclusion $K_{K_{0} \backslash\left\{v_{0}\right\}} \cup K_{K_{0} \backslash\left\{v_{1}\right\}} \subset K$.

There are two possibilities. First, $\left\{v_{0}, v_{1}\right\}$ is not a simplex in $K$. In this case $K_{K_{0} \backslash\left\{v_{0}\right\}} \cup K_{K_{0} \backslash\left\{v_{1}\right\}}=K$.

Assume $\left\{v_{0}, v_{1}\right\}$ is a simplex in $K$. Then:

$$
K=K_{K_{0} \backslash\left\{v_{0}\right\}} \cup K_{K_{0} \backslash\left\{v_{1}\right\}} \cup \operatorname{St}\left(v_{0}, v_{1}\right)
$$

Consequently there is a (homotopy) push-out square (see 4.5):

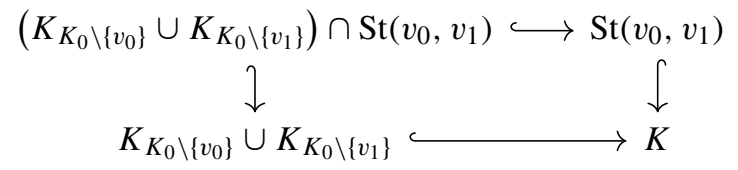

Since the star complex $\operatorname{St}\left(v_{0}, v_{1}\right)$ is contractible (see Proposition 4.9), $K$ is therefore weakly equivalent to the homotopy cofiber of the map:

$$
\left(K_{K_{0} \backslash\left\{v_{0}\right\}} \cup K_{K_{0} \backslash\left\{v_{1}\right\}}\right) \cap \operatorname{St}\left(v_{0}, v_{1}\right) \hookrightarrow K_{K_{0} \backslash\left\{v_{0}\right\}} \cup K_{K_{0} \backslash\left\{v_{1}\right\}}
$$

The complex $\left(K_{K_{0} \backslash\left\{v_{0}\right\}} \cup K_{K_{0} \backslash\left\{v_{1}\right\}}\right) \cap \operatorname{St}\left(v_{0}, v_{1}\right)$ fits into the following homotopy pushout square:

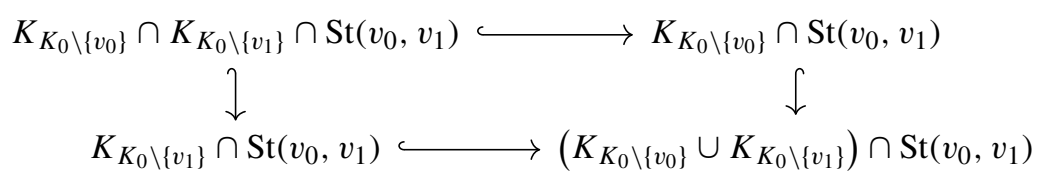

Let us identify the complexes in this square: 
$-K_{K_{0} \backslash\left\{v_{0}\right\}} \cap \operatorname{St}\left(v_{0}, v_{1}\right)=\left\{\mu \in K \mid \mu \cap\left\{v_{0}\right\}=\emptyset\right.$ and $\left.\left\{v_{0}, v_{1}\right\} \cup \mu \in K\right\}$ and thus according to Proposition 4.9 this complex is contractible;

- by the same argument $K_{K_{0} \backslash\left\{v_{1}\right\}} \cap \operatorname{St}\left(v_{0}, v_{1}\right)$ is also contractible;

- $K_{K_{0} \backslash\left\{v_{0}\right\}} \cap K_{K_{0} \backslash\left\{v_{1}\right\}} \cap \operatorname{St}\left(v_{0}, v_{1}\right)=K_{K_{0} \backslash\left\{v_{0}, v_{1}\right\}} \cap \operatorname{St}\left(v_{0}, v_{1}\right)=\operatorname{St}\left(\left\{v_{0}, v_{1}\right\}, K_{0} \backslash\left\{v_{0}, v_{1}\right\}\right)$

It follows that $\left(K_{K_{0} \backslash\left\{v_{0}\right\}} \cup K_{K_{0} \backslash\left\{v_{1}\right\}}\right) \cap \operatorname{St}\left(v_{0}, v_{1}\right)$ has the homotopy type of the suspension of the obstruction complex $\mathrm{St}:=\mathrm{St}\left(\left\{v_{0}, v_{1}\right\}, K_{0} \backslash\left\{v_{0}, v_{1}\right\}\right)$ and hence we have a homotopy cofiber sequence of the form:

$$
\Sigma \mathrm{St} \rightarrow K_{K_{0} \backslash\left\{v_{0}\right\}} \cup K_{K_{0} \backslash\left\{v_{1}\right\}} \hookrightarrow K
$$

\section{Several outside points}

Homotopy cofiber sequences as described in Sect. 5 and 6 are particular cases of a more general statement regarding an arbitrary number of outside points. The aim of this section is to present this generalization.

Let us fix a set $\sigma=\left\{v_{0}, v_{1}, \ldots, v_{n}\right\} \subset K_{0}$ of $n+1$ distinct vertices in a simplicial complex $K$ which may not necessarily be a simplex in $K$. Note that $\bigcup_{v \in \sigma}\left(K_{0} \backslash\{v\}\right)=$ $K_{0}$. In this section we are going to investigate the inclusion $\left(\bigcup_{v \in \sigma} K_{K_{0} \backslash\{v\}}\right) \subset K$

There are two possibilities. First, $\sigma$ is not a simplex in $K$. In this case $\bigcup_{v \in \sigma} K_{K_{0} \backslash\{v\}}=K$.

Assume $\sigma$ is a simplex in $K$. Then:

$$
K=\left(\bigcup_{v \in \sigma} K_{K_{0} \backslash\{v\}}\right) \cup \operatorname{St}(\sigma)
$$

Consequently there is a homotopy push-out square (see 4.5):

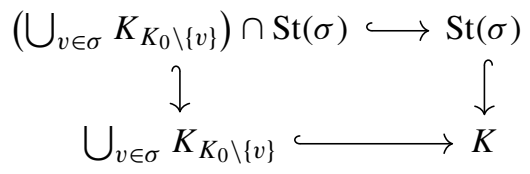

Since the star complex $\operatorname{St}(\sigma)$ is contractible (see Proposition 4.9), $K$ is therefore weakly equivalent to the homotopy cofiber of the map:

$$
\left(\bigcup_{v \in \sigma} K_{K_{0} \backslash\{v\}}\right) \cap \operatorname{St}(\sigma) \hookrightarrow \bigcup_{v \in \sigma} K_{K_{0} \backslash\{v\}}
$$

Next we identify the homotopy type of $\left(\bigcup_{v \in \sigma} K_{K_{0} \backslash\{v\}}\right) \cap \operatorname{St}(\sigma)$ :

Proposition 7.1 Let $\sigma$ be a simplex of dimension $n$ in a simplicial complex $K$. Then $\left(\bigcup_{v \in \sigma} K_{K_{0} \backslash\{v\}}\right) \cap S t(\sigma)$ has the homotopy type of the $n$-th suspension of the obstruction complex $\Sigma^{n} \operatorname{St}\left(\sigma, K_{0} \backslash \sigma\right)$. 
Proof Consider the inclusion poset of all subsets $\tau \subset \sigma$. For any such subset $\tau \subset \sigma$, define:

$$
F(\tau):= \begin{cases}\bigcap_{v \in \tau} K_{K_{0} \backslash\{v\}}=K_{K_{0} \backslash \tau} & \text { if } \tau \neq \emptyset \\ \bigcup_{v \in \sigma} K_{K_{0} \backslash\{v\}} & \text { if } \tau=\emptyset\end{cases}
$$

Note that if $\tau^{\prime} \subset \tau \subset \sigma$, then $F(\tau) \subset F\left(\tau^{\prime}\right)$. Thus by assigning to the inclusion $\tau^{\prime} \subset \tau$ the map $F(\tau) \subset F\left(\tau^{\prime}\right)$, we obtain a contravariant functor indexed by the inclusion poset of all subsets of $\sigma$. For example in the case $\sigma=\left\{v_{0}, v_{1}, v_{2}\right\}$, this contravariant functor describes a commutative cube:

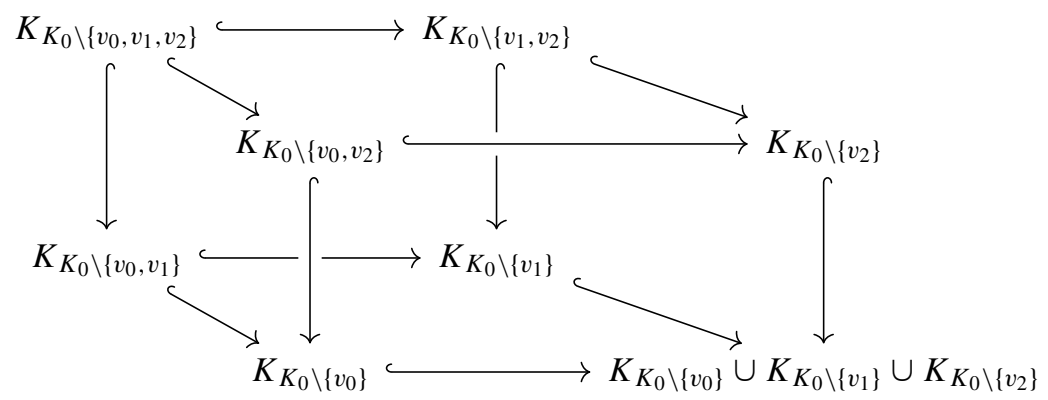

For arbitrary $n$, the functor $F$ describes a commutative cube of dimension $n+1$. This cube is both co-cartesian and strongly cartesian (Munson and Volic 2015). It is therefore also a homotopy co-cartesian. By intersecting with $\operatorname{St}(\sigma)$, we obtain a new cube $\tau \mapsto F(\tau) \cap \operatorname{St}(\sigma)$. The properties of being co-cartesian and strongly cartesian are preserved by taking such intersections. Consequently $\left(\bigcup_{v \in \sigma} K_{K_{0} \backslash\{v\}}\right) \cap \operatorname{St}(\sigma)$ has the homotopy type of hocolim $\emptyset \neq \tau \subset \sigma\left(K_{K_{0} \backslash \tau} \cap \operatorname{St}(\sigma)\right)$.

For any proper subset $\emptyset \neq \tau \subsetneq \sigma$, we have an equality:

$$
K_{K_{0} \backslash \tau} \cap \operatorname{St}(\sigma)=\{\mu \mid \mu \cap \tau=\emptyset \text { and } \sigma \cup \mu \in K\}
$$

We can then use Proposition 4.9 to conclude that $K_{K_{0} \backslash \tau} \cap \operatorname{St}(\sigma)$ is contractible if $\emptyset \neq \tau \subsetneq \sigma$. Thus all the spaces in the cube $\tau \mapsto F(\tau) \cap \operatorname{St}(\sigma)$, except for the initial and the terminal ones, are contractible. That implies that the terminal space $F(\emptyset) \cap \operatorname{St}(\sigma)=\left(\bigcup_{v \in \sigma} K_{K_{0} \backslash\{v\}}\right) \cap \operatorname{St}(\sigma)$ is homotopy equivalent to the $n$-th suspension of the initial space: $\Sigma^{n}(F(\sigma) \cap \operatorname{St}(\sigma))=\Sigma^{n}\left(K_{K_{0} \backslash \sigma} \cap \operatorname{St}(\sigma)\right)=\Sigma^{n} \operatorname{St}\left(\sigma, K_{0} \backslash \sigma\right)$.

We finish this section with summarising the consequences of the discussion leading to Proposition 7.1 and the proposition itself:

Corollary 7.2 Let $\sigma \subset K_{0}$ be a subset consisting of $n+1$ distinct vertices in a simplicial complex $K$.

1. If $\sigma$ is not a simplex in $K$, then $\bigcup_{v \in \sigma} K_{K_{0} \backslash\{v\}}=K$.

2. Assume $\sigma$ is a simplex in $K$. 
(a) Then there is a homotopy cofiber sequence:

$$
\Sigma^{n} \operatorname{St}\left(\sigma, K_{0} \backslash \sigma\right) \rightarrow \bigcup_{v \in \sigma} K_{K_{0} \backslash\{v\}} \hookrightarrow K
$$

(b) If $\operatorname{St}\left(\sigma, K_{0} \backslash \sigma\right)=\emptyset$, then there is a homotopy cofiber sequence (here $\left.S^{-1}=\emptyset\right)$ :

$$
S^{n-1} \rightarrow \bigcup_{v \in \sigma} K_{K_{0} \backslash\{v\}} \hookrightarrow K
$$

(c) If $\operatorname{St}\left(\sigma, K_{0} \backslash \sigma\right) \neq \varnothing$, then the homotopy fibers of $\bigcup_{v \in \sigma} K_{K_{0} \backslash\{v\}} \hookrightarrow K$ are $m$-connected for $m \geq 0$ if and only if $\bar{H}_{i}\left(\operatorname{St}\left(\sigma, K_{0} \backslash \sigma\right), \mathbf{Z}\right)=0$ for $i \leq m-n$.

(d) If $\operatorname{St}\left(\sigma, K_{0} \backslash \sigma\right) \neq \emptyset$, then $\bigcup_{v \in \sigma} K_{K_{0} \backslash\{v\}} \hookrightarrow K$ is a weak equivalence if and only if $\bar{H}_{i}\left(S t\left(\sigma, K_{0} \backslash \sigma\right), \mathbf{Z}\right)=0$ for all $i$.

\section{Push-out decompositions I}

In this section our starting assumption is:

8.1(Starting input I) $K$ is a simplicial complex, $X \cup Y=K_{0}$ is a cover of its set of vertices, and $A:=X \cap Y$.

By restricting $K$ to $X$ and $Y$, and taking the union of these restrictions we obtain a subcomplex $K_{X} \cup K_{Y} \subset K$. Since $K_{X} \cap K_{Y}=K_{A}$, this subcomplex fits into the following homotopy push-out square:

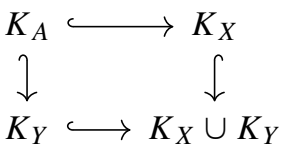

This push-out can be then used to extract various homotopical properties of the union $K_{X} \cup K_{Y}$ from the properties of $K_{X}, K_{Y}$ and $K_{A}$. For example, if $K_{X}, K_{Y}$ and $K_{A}$ belong to a closed collection (see 3.5), then so does $K_{X} \cup K_{Y}$. If $K_{A}$ is contractible, then $K_{X} \cup K_{Y}$ has the homotopy type of the wedge of $K_{X}$ and $K_{Y}$, and its reduced homology is the sum of the reduced homology of $K_{X}$ and $K_{Y}$. More generally, there is a Mayer-Vietoris sequence connecting homology of $K_{X} \cup K_{Y}$ with those of $K_{X}$, $K_{Y}$ and $K_{A}$.

A fundamental question discussed in this article is: under what circumstances is the inclusion $K_{X} \cup K_{Y} \subset K$ a weak equivalence, or homology isomorphism, or has highly connected homotopy fibers etc? Such circumstances would enable us to express various homotopical properties of $K$ in terms of the properties of its restrictions $K_{X}$, $K_{Y}$ and $K_{A}$.

Definition 8.2 Under the starting assumption 8.1, define $P$ to be the subposet of $K$ given by:

$$
P:=\{\sigma \in K \mid \sigma \subset X \text { or } \sigma \subset Y \text { or } \sigma \cap A \neq \emptyset\}
$$




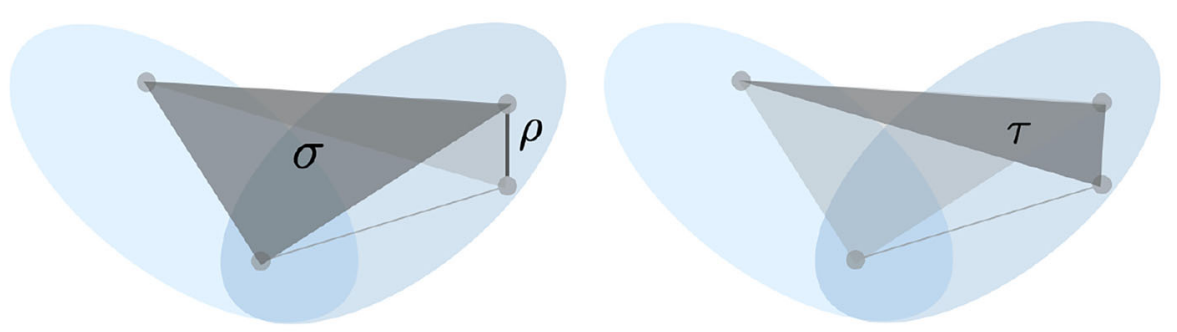

Fig. 2 The two light blue regions represent the restrictions $K_{X}$ and $K_{Y}$ of a simplicial complex $K$. The simplices $\sigma$ and $\rho$ belong to $P$ and $\tau$ is an example of a simplex in $K \backslash P$

Note that $P \cap \operatorname{sk}_{1} K=\operatorname{sk}_{1}\left(K_{X} \cup K_{Y}\right)$, in particular $K_{0} \subset P$.

We are going to be more interested in the set of simplices of $K$ that do not belong to $P$, which explicitly can be described as:

$$
K \backslash P=\{\sigma \in K \mid \sigma \cap X \neq \emptyset \text { and } \sigma \cap Y \neq \emptyset \text { and } \sigma \cap A=\emptyset\}
$$

The poset $P$ may not be the simplex category of any simplicial complex. There are two poset inclusions that we denote by $f$ and $g$ :

$$
K_{X} \cup K_{Y} \stackrel{f}{\longleftrightarrow} P \stackrel{g}{\hookrightarrow} K
$$

Our first general observation is:

Proposition 8.3 The functor $f: K_{X} \cup K_{Y} \hookrightarrow P$ is a weak equivalence.

Proof We are going to show that, for every $\sigma$ in $P, f \downarrow \sigma$ is contractible.

First assume $\sigma \subset X$ or $\sigma \subset Y$. Then the object $(\sigma, \mathrm{id}: \sigma \rightarrow \sigma)$ is terminal in $f \downarrow \sigma$, and consequently this category is contractible, and the proposition in this case follows from Corollary 3.8.1.

Assume $\sigma \cap A \neq \emptyset$. Then, for every object $(\tau, \tau \subset \sigma)$ in $f \downarrow \sigma$, the subsets $\tau$, $\tau \cup(\sigma \cap A)$, and $\sigma \cap A$ of $\sigma$ are simplices that belong to $K_{X} \cup K_{Y}$. We can then form the following commutative diagram in $P$ where the top horizontal arrows represent morphisms in $K_{X} \cup K_{Y}$ :

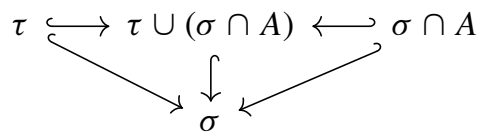

These horizontal morphisms form natural transformations between:

- the identity functor id: $f \downarrow \sigma \rightarrow f \downarrow \sigma,(\tau, \tau \subset \sigma) \mapsto(\tau, \tau \subset \sigma)$,

- the constant functor $f \downarrow \sigma \rightarrow f \downarrow \sigma,(\tau, \tau \subset \sigma) \mapsto(\sigma \cap A, \sigma \cap A \subset \sigma)$,

- and $f \downarrow \sigma \rightarrow f \downarrow \sigma$ given by $(\tau, \tau \subset \sigma) \mapsto(\tau \cup(\sigma \cap A), \tau \cup(\sigma \cap A) \subset \sigma)$. 
The identity functor id: $f \downarrow \sigma \rightarrow f \downarrow \sigma$ is therefore homotopic to the constant functor. This can happen only if $f \downarrow \sigma$ is a contractible category.

The dimensions of the simplices involved in the constructions performed in the proof of Proposition 8.3 do not exceed the dimension of the considered simplex $\sigma$. Consequently, this proof shows a stronger statement:

Proposition 8.4 The functor $f:\left(K_{X} \cup K_{Y}\right) \cap s k_{n} K \hookrightarrow P \cap s k_{n} K$ is a weak equivalence for every natural number $n$.

According to Proposition 8.3, the homotopy fibers of $g: P \subset K$ and the inclusion $K_{X} \cup K_{Y} \subset K$ are weakly equivalent. To understand these homotopy fibers, we are going to focus on the categories $\sigma \uparrow g$ and then utilise Corollary 3.8. The functor $\sigma \mapsto \sigma \uparrow g$ fits into the following diagram of natural transformations between functors indexed by $K^{\text {op }}$ with small categories as values:

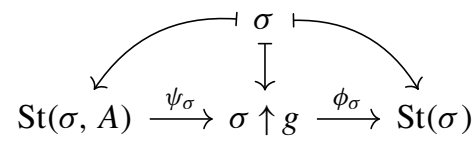

where:

- $\psi_{\sigma}: \operatorname{St}(\sigma, A) \rightarrow \sigma \uparrow g$ assigns to $\mu$ in $\operatorname{St}(\sigma, A)$ the object in $\sigma \uparrow g$ given by the pair $\psi_{\sigma}(\mu):=(\mu \cup \sigma, \sigma \subset \mu \cup \sigma)$.

$-\phi_{\sigma}: \sigma \uparrow g \rightarrow \operatorname{St}(\sigma)$ assigns to $(\tau, \sigma \subset \tau)$ the simplex $\tau$ in $\operatorname{St}(\sigma)$.

The contravariant functor $\sigma \mapsto \sigma \uparrow g$ interpolates between the contravariant functors $\sigma \mapsto \operatorname{St}(\sigma)$ (see 4.8) and $\sigma \mapsto \operatorname{St}(\sigma, A)$ (see 5.1) in the following sense:

Proposition 8.5 Let $\sigma$ be a simplex in $K$.

1. If $\sigma$ is in $P$, then $\sigma \uparrow g$ is contractible and $\phi_{\sigma}: \sigma \uparrow g \rightarrow$ St $(\sigma)$ is a weak equivalence.

2. If $\sigma$ is in $K \backslash P$, then $\psi_{\sigma}: \operatorname{St}(\sigma, A) \rightarrow \sigma \uparrow g$ is a weak equivalence.

Proof If $\sigma$ is in $P$, then ( $\sigma$, id: $\sigma \rightarrow \sigma)$ is an initial object in $\sigma \uparrow g$ and hence this category is contractible. That proves (1).

Assume $\sigma$ is not in $P$, which is equivalent to $\sigma \cap Y \neq \emptyset$ and $\sigma \cap X \neq \emptyset$ and $\sigma \cap A=\emptyset$. Let $(\tau, \sigma \subset \tau)$ be an object in $\sigma \uparrow g$. Define $\alpha_{\sigma}(\tau, \sigma \subset \tau):=\tau \cap A$. Since $\sigma \cap Y \neq \emptyset$ and $\sigma \cap X \neq \emptyset$, then $\tau \cap Y \neq \emptyset$ and $\tau \cap X \neq \emptyset$. This together with the fact that $\tau$ belongs to $P$ implies $\alpha_{\sigma}(\tau, \sigma \subset \tau)=\tau \cap A \neq \emptyset$. Furthermore $(\tau \cap A) \cup \sigma \subset \tau \in P \subset K$. Thus $\alpha_{\sigma}$ defines a functor $\alpha_{\sigma}: \sigma \uparrow g \rightarrow \operatorname{St}(\sigma, A)$. Note:

$$
\alpha_{\sigma} \psi_{\sigma}(\mu)=\alpha_{\sigma}(\mu \cup \sigma, \sigma \subset \mu \cup \sigma)=(\mu \cup \sigma) \cap A
$$

Since $\sigma \cap A=\emptyset$ and $\mu \subset A$, we get $\alpha_{\sigma} \psi_{\sigma}(\mu)=(\mu \cup \sigma) \cap A=\mu$. The composition $\alpha_{\sigma} \psi_{\sigma}$ is therefore the identity functor.

Note further:

$$
\psi_{\sigma} \alpha_{\sigma}(\tau, \sigma \subset \tau)=\psi_{\sigma}(\tau \cap A)=((\tau \cap A) \cup \sigma, \sigma \subset(\tau \cap A) \cup \sigma)
$$


Since $\sigma \subset \tau$, we have a commutative diagram:

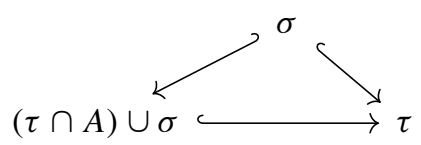

The bottom horizontal morphisms form a natural transformation between:

- the composition $\psi_{\sigma} \alpha_{\sigma}: \sigma \uparrow g \rightarrow \sigma \uparrow g$ and

- the identity functor id: $\sigma \uparrow g \rightarrow \sigma \uparrow g$.

The functor $\psi_{\sigma}: \operatorname{St}(\sigma, A) \rightarrow \sigma \uparrow g$ has therefore a homotopy inverse and hence is a weak equivalence which proves (2).

We use Corollary 3.8 and Proposition 8.5 to obtain our main statement describing properties of the homotopy fibers of the inclusion $K_{X} \cup K_{Y} \subset K$ :

Theorem 8.6 Notation as in 8.1 and Definition 8.2. Let $\mathcal{C}$ be a closed collection of simplicial sets (see 3.5). Assume that, for every $\sigma$ in $K \backslash P$, the obstruction complex $\operatorname{St}(\sigma, A)$ (see 5.1) satisfies $\mathcal{C}$. Then the homotopy fibers of the inclusion $K_{X} \cup K_{Y} \subset K$ also satisfy $\mathcal{C}$.

The following are some particular cases of the above theorem specialized to different closed collections of simplicial sets.

Corollary 8.7 Notation as in 8.1 and 8.2. Let $n$ be a natural number.

1. If, for every $\sigma$ in $K \backslash P$ (see 8.2), the simplicial complex $\operatorname{St}(\sigma, A)$ (see 5.1) is contractible, then $K_{X} \cup K_{Y} \subset K$ is a weak equivalence.

2. If, for every $\sigma$ in $K \backslash P$, the simplicial complex $\operatorname{St}(\sigma, A)$ is $n$-connected, then the homotopy fibers of $K_{X} \cup K_{Y} \subset K$ are $n$-connected and this map induces an isomorphism on homotopy groups in degrees $0, \ldots, n$ and a surjection in degree $n+1$.

3. Let $p$ be a prime number. If, for every $\sigma$ in $K \backslash P$, the simplicial complex $\operatorname{St}(\sigma, A)$ is connected and has p-torsion reduced integral homology in degrees not exceeding $n$, then the homotopy fibers of $K_{X} \cup K_{Y} \subset K$ are connected and have $p$-torsion reduced integral homology in degrees not exceeding $n$. Thus in this case, for prime $q \neq p, K_{X} \cup K_{Y} \subset K$ induces an isomorphism on $H_{*}(-, \mathbf{Z} / q)$ for $* \leq n$ and $a$ surjection on $H_{n+1}(-, \mathbf{Z} / q)$.

4. If, for every $\sigma$ in $K \backslash P$, the simplicial complex $S t(\sigma, A)$ is acyclic with respect to some homology theory, then $K_{X} \cup K_{Y} \subset K$ is this homology isomorphism.

Requirements for obtaining $n$-connected fibers can be weakened:

Proposition 8.8 Notation as in 8.1 and 8.2. Let $n$ be a natural number. If $\operatorname{St}(\sigma, A)$ is $n$-connected for every $\sigma$ in $\left(s k_{n+1} K\right) \backslash P$, then the homotopy fibers of $K_{X} \cup K_{Y} \subset K$ are $n$-connected. 
Proof For every $m \geq n+1$ Consider the following poset inclusions:

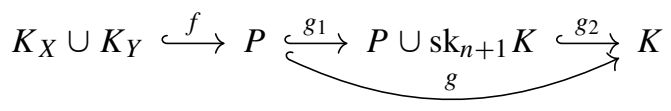

According to Proposition 8.3, $f$ is a weak equivalence. The homotopy fibers of $g_{2}$ are $n$-connected by Proposition 4.7. Thus if the homotopy fibers of $g_{1}$ are $n$-connected, then so are the homotopy fibers of the inclusion $K_{X} \cup K_{Y} \subset K$. To show that the homotopy fibers of $g_{1}$ are $n$-connected it is enough to show that the categories $\sigma \uparrow g_{1}$ are $n$-connected for every $\sigma$ in $P \cup \mathrm{sk}_{n+1} K$. Proposition 8.5 gives that $\sigma \uparrow g_{1}$ is contractible if $\sigma$ is in $P$, and is weakly equivalent to $\operatorname{St}(\sigma, A)$ if $\sigma$ is in $\operatorname{sk}_{n+1} K \backslash P$. By the assumption $\operatorname{St}(\sigma, A)$ are therefore $n$-connected.

It would be natural at this point to wonder about a generalisation of our results to coverings of the set of vertices of a simplicial complex $K$ by more than two sets. In that case, any possible intersection between sets of the considered covering should be taken into account. This not only increases the level of complexity of the problem, but also makes it very easy to lose control over the information one aims to obtain, since some of the intersections may be empty. Although much more complex, the situation is not hopeless. For example, in Sect. 7 we already considered instances of such coverings for which a decomposition statement holds.

\section{Push-out decompositions II}

Theorem 8.6 states that the homotopy fibers of the inclusion $K_{X} \cup K_{Y} \subset K$ belong to the smallest closed collection containing all the complexes $\operatorname{St}(\sigma, A)$ for $\sigma$ in $K \backslash P$. Recall that if a closed collection contains an empty simplicial set, then it contains all simplicial sets, in which case Theorem 8.6 has no content. Thus $\operatorname{St}(\sigma, A)$ being non empty, for all $\sigma$ in $K \backslash P$, is an absolute minimum requirement for Theorem 8.6 to have any content. In most of our statements that follow, the assumptions we make have much stronger global non emptiness consequences of the form:

$$
\bigcap_{\sigma \in K \backslash P} \operatorname{St}(\sigma, A) \neq \emptyset \quad \bigcap_{\sigma \in K_{n+1} \backslash P} \operatorname{St}(\sigma, A) \neq \emptyset \quad \bigcap_{\sigma \in\left(\mathrm{sk}_{n+1} K\right) \backslash P} \operatorname{St}(\sigma, A) \neq \emptyset
$$

Here is a consequence of having one of these intersections non-empty:

Proposition 9.1 Notation as in 8.1 and 8.2. Assume:

$$
\bigcap_{\sigma \in K_{1} \backslash P} \operatorname{St}(\sigma, A) \neq \emptyset
$$

Then the homotopy fibers of $K_{X} \cup K_{Y} \subset K$ are connected. 
Proof Let $v$ be a vertex in $\bigcap_{\sigma \in K_{1} \backslash P} \operatorname{St}(\sigma, A)$. Observe that $\operatorname{sk}_{1}(K)$ is a disjoint union of $K_{1} \backslash P$ and $\operatorname{sk}_{1}(K) \cap P$. This can fail for $\operatorname{sk}_{n}(K)$ if $n>1$. For every $\tau$ in $\operatorname{sk}_{1}(K)$, define:

$$
\phi(\tau):= \begin{cases}\tau \cup v & \text { if } \tau \in K_{1} \backslash P \\ \tau & \text { if } \tau \in \operatorname{sk}_{1}(K) \cap P\end{cases}
$$

Since $v$ is in $A$, we have $(\tau \cup\{v\}) \cap A \neq \emptyset$, and hence $\phi(\tau)$ belongs to $P$. If $\tau \subsetneq \tau^{\prime}$ in $\operatorname{sk}_{1}(K)$, then $\tau$ is in $P$ and hence $\tau=\phi(\tau) \subset \phi\left(\tau^{\prime}\right)$. In this way we obtain a functor $\phi: \operatorname{sk}_{1}(K) \rightarrow P$. The inclusion $\tau \subset \phi(\tau)$, is a natural transformation between the skeleton inclusion $\operatorname{sk}_{1}(K) \subset K$ and the composition:

$$
\operatorname{sk}_{1}(K) \stackrel{\phi}{\longrightarrow} P \stackrel{g}{\longrightarrow} K
$$

Thus these two functors from $\operatorname{sk}_{1}(K)$ to $K$ are homotopic. The map $g: P \hookrightarrow K$ and, hence the inclusion $K_{X} \cup K_{Y} \subset K$, induces therefore a surjection on $\pi_{1}$ for every choice of a basepoint. Thus to show $K_{X} \cup K_{Y} \subset K$ has connected homotopy fibers it is enough to prove this map induces a bijection on $\pi_{0}$. Surjection on $\pi_{0}$ is clear. Let $\tau_{0}, \ldots, \tau_{n}$ be a path of edges in $K$ connecting two vertices. If an edge $\tau_{i}=\{x, y\}$ in this path does not belong to $K_{X} \cup K_{Y}$, then it can not belong to $P$ either. In this case $\{x, y, v\}$ is a simplex in $K$, and we can substitute $\tau_{i}$, in the considered path, by two edges $\{x, v\},\{v, y\}$. By performing these substitutions we obtain a path consisting only of edges in $K_{X} \cup K_{Y}$. The map $K_{X} \cup K_{Y} \subset K$ induces therefore an injection on $\pi_{0}$.

Proposition 9.1 does not generalise to $n>0$. Non-emptiness of the intersection $\bigcap_{\sigma \in\left(\mathrm{sk}_{n+1} K\right) \backslash P} \operatorname{St}(\sigma, A)$ does not imply that the homotopy fibers of $K_{X} \cup K_{Y} \subset K$ are $n$-connected. For an easy example see 11.4 . To guarantee $n$-connectedness of these homotopy fibers we need additional restrictions. For example in the following corollary the assumptions imply that $\operatorname{St}(\sigma, A)$ does not depend on $\sigma$ in $\left(\operatorname{sk}_{n+1} K\right) \backslash P$ :

Corollary 9.2 Notation as in 8.1 and 8.2. Let $n$ be a natural number. Assume that one of the following conditions is satisfied:

1. There is an n-connected simplicial complex $L$ such that, for every simplex $\sigma$ in $\left(s k_{n+1} K\right) \backslash P, S t(\sigma, A)=L$.

2. The complex $K_{A}$ is $n$-connected and, for every simplex $\sigma$ in $\left(s k_{n+1} K\right) \backslash P$, $\operatorname{St}(\sigma, A)=K_{A}$.

3. The set $A$ is non empty. Furthermore, for every simplex $\sigma$ in $\left(s k_{n+1} K\right) \backslash P$ and every finite subset $\mu$ in $A$, the union $\sigma \cup \mu$ is a simplex in $K$.

4. $A=\{v\}$ and, for every simplex $\sigma$ in $\left(s k_{n+1} K\right) \backslash P$, the union $\sigma \cup\{v\}$ is also a simplex in $K$.

Then the homotopy fibers of $K_{X} \cup K_{Y} \subset K$ are n-connected.

Proof The corollary under assumption 1 is a direct consequence of Proposition 8.8. Assumption 2 is a particular case of 1 with $L=K_{A}$. Assumption 3 is a particular case of 1 with $L=\Delta[A]$. Finally, assumption 4 is a particular case of 3 . 
Here is another example of a statement whose assumption, referred to as "one entry point", has a global nonemptiness consequence:

Corollary 9.3 Notation as in 8.1 and 8.2. Let $n$ be a natural number. Assume there is an element $v$ in $A$ with the following property. For every simplex $\tau$ in $K$ such that $\tau \cap(X \backslash A) \neq \emptyset, \tau \cap(Y \backslash A) \neq \emptyset$, and $\left|\tau \cap\left(K_{0} \backslash A\right)\right| \leq n+2$, the union $\tau \cup\{v\}$ is also a simplex in $K$. Then, for every simplex $\sigma$ in $\left(s k_{n+1} K\right) \backslash P$, the element $v$ is a central vertex (see 4.10) in $\operatorname{St}(\sigma, A)$. Furthermore the homotopy fibers of $K_{X} \cup K_{Y} \subset K$ are n-connected.

Proof Let $\sigma$ be a simplex in $\left(\operatorname{sk}_{n+1} K\right) \backslash P$. Thus $\sigma \cap(X \backslash A) \neq \emptyset, \sigma \cap(Y \backslash A) \neq \emptyset$, and $\left|\sigma \cap\left(K_{0} \backslash A\right)\right|=|\sigma| \leq n+2$. If $\mu$ belongs to $\operatorname{St}(\sigma, A)$ then, $\tau=\sigma \cup \mu$ also satisfies these assumptions, since $\mu$ is entirely contained in $A$, and the hypothesis of the corollary only involve points outside $A$. Therefore $\sigma \cup \mu \cup\{v\}$ is a simplex in $K$ and hence $\mu \cup\{v\}$ is a simplex in $\operatorname{St}(\sigma, A)$. This means $v$ is central in $\operatorname{St}(\sigma, A)$ (see 4.10). Consequently, $\operatorname{St}(\sigma, A)$ is contractible (see 4.10) and the corollary follows from Proposition 8.8 .

\section{Clique complexes}

Recall that a simplicial complex $K$ is called clique if it satisfies the following condition: a set $\sigma$ of size at least 2 is a simplex in $K$ if and only if all the two element subsets of $\sigma$ are simplices in $K$. Thus a clique complex is determined by its sets of vertices and edges.

If $K$ is clique, then the complexes $\operatorname{St}(\sigma, A)$ satisfy the following properties:

Proposition 10.1 Notation as in 8.1. Assume $K$ is clique. Then:

1. For all $\sigma$ in $K, \operatorname{St}(\sigma, A)$ is clique.

2. If $\tau$ and $\sigma$ are simplices in $K$ such that $\tau \cup \sigma$ is also a simplex in $K$, then $\operatorname{St} \tau \cup$ $\sigma, A)=\operatorname{St}(\tau, A) \cap \operatorname{St}(\sigma, A)$.

3. If $\sigma$ is a simplex in $K$ and $\sigma=\tau_{1} \cup \cdots \cup \tau_{n}$, then $\operatorname{St}(\sigma, A)=\bigcap_{i=1}^{n} \operatorname{St}\left(\tau_{i}, A\right)$.

4. For every simplex $\sigma$ in $K, \operatorname{St}(\sigma, A)=\bigcap_{x \in \sigma} \operatorname{St}(\{x\}, A)$.

Proof Let $\mu$ be a subset of $A$ such that, for every two element subset $\tau$ of $\mu$, the set $\tau \cup \sigma$ is a simplex in $K$, i.e., $\tau$ is in $\operatorname{St}(\sigma, A)$. Then, since $K$ is clique, $\mu \cup \sigma$ is also a simplex in $K$. Consequently, $\mu$ belongs to $\operatorname{St}(\sigma, A)$ and hence $\operatorname{St}(\sigma, A)$ is clique. That proves (1).

To prove (2), first note that the inclusion $\operatorname{St}(\tau \cup \sigma, A) \subset \operatorname{St}(\tau, A) \cap \operatorname{St}(\sigma, A)$ holds even without the clique assumption. Let $\mu$ belong to both $\operatorname{St}(\tau, A)$ and $\operatorname{St}(\sigma, A)$. This means that $\mu \cup \tau$ and $\mu \cup \sigma$ are simplices in $K$. Since every 2 element subset of $\mu \cup \tau \cup \sigma$ is a subset of either $\mu \cup \tau$ or $\mu \cup \sigma$ or $\tau \cup \sigma$, by the assumption it is an edge in $K$. By the clique assumption, $\mu \cup \tau \cup \sigma$ is then also a simplex in $K$ and consequently $\mu$ is in $\operatorname{St}(\tau \cup \sigma, A)$. This shows the other inclusion $\operatorname{St}(\tau \cup \sigma, A) \supset \operatorname{St}(\tau, A) \cap \operatorname{St}(\sigma, A)$ proving (2).

Statements (3) and (4) follow from (2). 
The clique assumption in Proposition 10.1 is essential. For example consider the situation described in Fig. 1 where the complex is not clique. In this example $\operatorname{St}\left(v_{0}, A\right)=\left\{a_{0}\right\}$ and $\operatorname{St}\left(v_{1}, A\right)=\left\{a_{1}\right\}$, so $\operatorname{St}\left(v_{0}, A\right) \cap \operatorname{St}\left(v_{1}, A\right)=\emptyset$, while $\operatorname{St}(\sigma, A)=\left\{a_{0}\right\} \cup\left\{a_{1}\right\}$.

Recall that an intersection of standard simplices is again a standard simplex (see 4.3). This observation together with Propositions 8.8 and 10.1 gives:

Corollary 10.2 Notation as in 8.1 and 8.2. Assume $K$ is clique and, for every edge $\tau$ in $K_{1} \backslash P$, the complex $\operatorname{St}(\tau, A)$ is a standard simplex. If, for all simplices $\sigma$ in $\left(s k_{n+1} K\right) \backslash P$, the complex $\operatorname{St}(\sigma, A)$ is non-empty, then the homotopy fibers of the inclusion $K_{X} \cup K_{Y} \hookrightarrow K$ are $n$-connected.

Since clique complexes are determined by their edges, one can wonder if, for such complexes, the conclusions of Corollaries 9.2 and 9.3 would still hold true if their assumptions are verified only for low dimensional simplices. Here is an analogue of Corollary 9.2 for clique complexes.

Proposition 10.3 Notation as in 8.1 and 8.2. Let $n$ be a natural number. Assume $K$ is clique and that one of the following conditions is satisfied:

1. There is an n-connected simplicial complex $L$ such that, for every edge $\tau$ in $K_{1} \backslash P$, $\operatorname{St}(\tau, A)=L$.

2. The complex $K_{A}$ is $n$-connected and, for every edge $\tau$ in $K_{1} \backslash P$ and every element $v$ in $A$, the set $\tau \cup\{v\}$ is a simplex in $K$.

Then the homotopy fibers of the inclusion $K_{X} \cup K_{Y} \hookrightarrow K$ are $n$-connected.

Proof Assumption 1 together with Proposition 10.1.4 implies assumption 1 of Corollary 9.2, proving the proposition in this case.

Let $\tau$ be an edge in $K_{1} \backslash P$ and $\mu$ be a simplex in $K_{A}$. Assume (2). This assumption implies that any two element subset of $\tau \cup \mu$ is a simplex in $K$. Since $K$ is clique, the set $\tau \cup \mu$ is a simplex in $K$ and consequently $\mu$ is a simplex in $\operatorname{St}(\tau, A)$. Thus for any $\tau$ in $K_{1} \backslash P$, there is an inclusion $K_{A} \subset \operatorname{St}(\tau, A)$, and hence $K_{A}=\operatorname{St}(\tau, A)$ for any such $\tau$. Assumption 2 implies therefore assumption 1 with $L=K_{A}$.

Corollary 10.4 Notation as in 8.1 and 8.2. Assume $K$ is clique and that one of the following conditions is satisfied:

1. The set $A$ is non empty. Furthermore, for every edge $\tau$ in $K_{1} \backslash P$ and every subset $\mu$ in A such that $|\mu| \leq 2$, the union $\tau \cup \mu$ is a simplex in $K$.

2. $A=\{v\}$ and, for every edge $\tau$ in $K_{1} \backslash P$, the set $\tau \cup\{v\}$ is also a simplex in $K$.

Then the inclusion $K_{X} \cup K_{Y} \hookrightarrow K$ is a weak equivalence.

Proof Assume (1). If $K_{1} \backslash P$ is empty, then so is $K \backslash P$, and hence $P=K$. In this case the corollary follows from Proposition 8.3. Assume $K_{1} \backslash P$ is non-empty. Since any two element subset of $A$ is a simplex in $K$ and $K$ is clique, then all finite non-empty subsets of $A$ belong to $K$ and hence $K_{A}=\Delta[A]$. In this case assumption 1 is a particular case of condition 2 in Proposition 10.3 for all $n$ as $\Delta[A]$ is contractible.

Finally note that assumption 2 is a particular case of 1 . 
The following is an analogue of Corollary 9.3 which is also referred to as "one entry point".

Proposition 10.5 Notation as in 8.1 and 8.2. Assume $K$ is clique and that one of the following conditions is satisfied:

1. There is a vertex $v$ in $\bigcap_{\tau \in K_{1} \backslash P} S t(\tau, A)$ such that, for every edge $\tau$ in $K_{1} \backslash P$ and every vertex $w$ in $\operatorname{St}(\tau, A),\{v, w\}$ is a simplex in $K$.

2. There is a vertex $v$ in $\bigcap_{\tau \in K_{1} \backslash P} S t(\tau, A)$ such that, for every edge $\tau$ in $K_{1} \backslash P$, $v$ is a central vertex of $\operatorname{St}(\tau, A)$ (see 4.10).

3. There is an element $v$ in $A$ with the following property. For every simplex $\tau$ in $K$ such that $|\tau \cap(X \backslash A)|=1,|\tau \cap(Y \backslash A)|=1$, and $|\tau \cap A| \leq 1$, the union $\tau \cup\{v\}$ is also a simplex in $K$.

Then the inclusion $K_{X} \cup K_{Y} \hookrightarrow K$ is a weak equivalence.

Proof Assume 1. We aim to show $\operatorname{St}(\sigma, A)$ is contractible and then conclude by applying Corollary 8.7.1. Let $\sigma$ be a simplex in $K \backslash P$. Choose a cover $\sigma=\tau_{1} \cup \cdots \cup \tau_{n}$ where $\tau_{i}$ is an edge in $K_{1} \backslash P$ for all $i$. Then according to Proposition 10.1.3, $\operatorname{St}(\sigma, A)=$ $\bigcap_{i=1}^{n} \operatorname{St}\left(\tau_{i}, A\right)$. Let $w$ be a vertex in $\operatorname{St}(\sigma, A)$. Then it is also a vertex $\operatorname{in} \operatorname{St}\left(\tau_{i}, A\right)$ for all $i$. By the assumption $\{v, w\}$ is then a simplex in $K$. Thus all the 2 element subsets of $\sigma \cup\{v, w\}$ are simplices in $K$ and hence $\{v, w\}$ is a simplex in $\operatorname{St}(\sigma, A)$. As this happens for all vertices $w$ in $\operatorname{St}(\sigma, A)$, since $\operatorname{St}(\sigma, A)$ is clique, for every simplex $\mu$ in $\operatorname{St}(\sigma, A)$, the set $\mu \cup\{v\}$ is also a simplex in $\operatorname{St}(\sigma, A)$. The vertex $v$ is therefore central in $\operatorname{St}(\sigma, A)$ and consequently $\operatorname{St}(\sigma, A)$ is contractible. The proposition under assumption 1 follows then from Corollary 8.7.1.

Condition 2 is a particular case of 1 .

Assume 3. Let $\tau$ be an edge in $K_{1} \backslash P$. This in particular means $|\tau \cap A|=0$. Condition 3, applied to the simplex $\tau$, gives that $\tau \cup\{v\}$ is a simplex in $K$, and hence $v$ is a vertex in $\operatorname{St}(\tau, A)$. Let $w$ be a vertex in $\operatorname{St}(\tau, A)$. Then $|(\tau \cup\{w\}) \cap A|=1$ and by applying condition 3 to the simplex $\tau \cup\{w\}$ we get that $\{v, w\} \subset \tau \cup\{v, w\}$ are simplices in $K$. We can conclude that 3 implies 1 .

We finish this section with a statement referred to as "two entry points". This has been inspired by Adamaszek et al. (2020, Theorem 3), in which the gluing of two metric graphs along a path is considered. While in that case the two entry points are the endpoints of the path the graphs are glued along, in our framework they have to satisfy the listed properties. In both cases however this couple of points determine the weak equivalence stated.

Proposition 10.6 Notation as in 8.1 and 8.2. Assume $K$ is clique and there are two elements $a_{X}$ and $a_{Y}$ in $A$ with the following properties:

- For everyedge $\tau$ in $K_{1}$ such that $|\tau \cap A|=1$ and $|\tau \cap(X \backslash A)|=1$, the set $\tau \cup\left\{a_{X}\right\}$ is a simplex in $K$.

- For every edge $\tau$ in $K_{1}$ such that $|\tau \cap A|=1$ and $|\tau \cap(Y \backslash A)|=1$, the set $\tau \cup\left\{a_{Y}\right\}$ is a simplex in $K$.

- For every edge $\tau$ in $K_{1} \backslash P$, the set $\tau \cup\left\{a_{X}, a_{Y}\right\}$ is a simplex in $K$. Then, for every $\sigma$ in $K \backslash P$, the set $\left\{a_{X}, a_{Y}\right\}$ is a central simplex (see 4.10) in $\operatorname{St}(\sigma, A)$, and the inclusion $K_{X} \cup K_{Y} \subset K$ is a weak equivalence. 
Proof Let $\sigma$ be a simplex in $K \backslash P$. Any vertex $v$ in $\sigma$ is a vertex of an edge $\tau \subset \sigma$ that belongs to $K_{1} \backslash P$. According to the assumption, the sets $\left\{v, a_{X}, a_{Y}\right\} \subset \tau \cup\left\{a_{X}, a_{Y}\right\}$ are simplices in $K$. This, together with the clique assumption on $K$, implies $\sigma \cup\left\{a_{X}, a_{Y}\right\}$ is a simplex in $K$. Consequently, $\left\{a_{X}, a_{Y}\right\}$ is a simplex in $\operatorname{St}(\sigma, A)$.

Let $\mu$ be a simplex in $\operatorname{St}(\sigma, A)$. To prove the proposition, we need to show the set $\mu \cup\left\{a_{X}, a_{Y}\right\}$ is a simplex in $\operatorname{St}(\sigma, A)$ or equivalently $\sigma \cup \mu \cup\left\{a_{X}, a_{Y}\right\}$ is a simplex in $K$. Let $x$ be an arbitrary element in $\sigma \cap X, y$ an arbitrary element in $\sigma \cap Y$, and $v$ an arbitrary element in $\mu$. The sets $\{x, v\},\{y, v\}$, and $\{x, y\}$ are simplices in $K$. Thus according to the assumptions so are $\left\{x, v, a_{X}\right\},\left\{y, v, a_{Y}\right\}$, and $\left\{x, y, a_{X}, a_{Y}\right\}$. Consequently, the 2-element sets $\left\{x, a_{X}\right\},\left\{v, a_{X}\right\},\left\{y, a_{X}\right\},\left\{x, a_{Y}\right\},\left\{v, a_{Y}\right\},\left\{y, a_{Y}\right\}$, $\left\{a_{X}, a_{Y}\right\},\{x, y\}$ are simplices in $K$. Since all the 2-element subsets of $\sigma \cup \mu \cup\left\{a_{X}, a_{Y}\right\}$ are of such a form and $K$ is clique, $\sigma \cup \mu \cup\left\{a_{X}, a_{Y}\right\}$ is a simplex in $K$.

\section{Vietoris-Rips complexes for distances}

Let $Z$ be a subset of the universe $\mathcal{U}$ (see 4.1). A function $d: Z \times Z \rightarrow[0, \infty]$ is called a distance if it is symmetric $d(x, y)=d(y, x)$ and reflexive $d(x, x)=0$ for all $x$ and $y$ in $Z$. A pair $(Z, d)$ is called a distance space. A distance space $(Z, d)$ is sometimes denoted simply by $Z$, if $d$ is understood from the context, or by $d$, if $Z$ is understood from the context.

Let $(Z, d)$ be a distance space. The diameter of a non empty and finite subset $\sigma \subset Z$ is by definition $\operatorname{diam}(\sigma):=\max \{d(x, y) \mid x, y \in \sigma\}$.

A subset $X \subset Z$ together with the distance function given by the restriction of $d$ to $X$ is called a subspace of $(Z, d)$.

Let $(Z, d)$ be a distance space and $r$ be in $[0, \infty)$. By definition, the VietorisRips complex $\operatorname{VR}_{r}(Z)$ consists of these non-empty finite subsets $\sigma \subset Z$ for which $\operatorname{diam}(\sigma) \leq r$ (explicitly: $d(x, y) \leq r$ for all $x$ and $y$ in $\sigma$ ). Vietoris-Rips complexes are examples of clique complexes (see Sect. 10).

Let $X$ be a subspace of $(Z, d)$. Then the Vietoris-Rips complex $\operatorname{VR}_{r}(X)$ coincides with the restriction $\operatorname{VR}_{r}(Z)_{X}$ (see 4.3).

Our starting assumption in this section is:

11.1 (Starting input II) $(Z, d)$ is a distance space, $X \cup Y=Z$ is a cover of $Z$, and $A:=X \cap Y$.

In the rest of this section we are going to reformulate in terms of the distance $d$ on $Z$ some of the statements given in the previous sections regarding the homotopy properties of the inclusion $\operatorname{VR}_{r}(X) \cup \mathrm{VR}_{r}(Y) \hookrightarrow \mathrm{VR}_{r}(Z)$ for various $r$ in $[0, \infty)$. Here is a direct restatement of Proposition 10.3:

Proposition 11.2 Notation as in 11.1. Let $r$ be an element in $[0, \infty)$ and $n$ be a natural number. Assume that one of the following conditions is satisfied:

1. There is a subset $L \subset A$ such that $V R_{r}(L)$ is n-connected and, for every $x$ in $X \backslash A$ and every $y$ in $Y \backslash A$ with $d(x, y) \leq r$, there is an equality $\{v \in A \mid d(x, v) \leq$ $r$ and $d(y, v) \leq r\}=L$, in particular the set on the left does not depend on $x$ and $y$. 
2. The complex $V R_{r}(A)$ is n-connected and, for all $x$ in $X \backslash A$, $y$ in $Y \backslash A$, and $v$ in $A$, if $d(x, y) \leq r$, then both $d(x, v) \leq r$ and $d(v, y) \leq r$.

Then the homotopy fibers of the inclusion $V R_{r}(X) \cup V R_{r}(Y) \subset V R_{r}(Z)$ are $n$ connected.

Assumption 2 of Proposition 11.2 can be restated as: (connectivity condition) $\mathrm{VR}_{r}(A)$ is $n$-connected, and (intersection condition) if $\mathrm{VR}_{r}(Z)_{1} \backslash P$ (see 8.2 for the definition of $P$ ) is non-empty, then

$$
A=\bigcap_{\sigma \in \mathrm{VR}_{r}(Z)_{1} \backslash P} \operatorname{St}(\sigma, A)_{0}
$$

What if the intersection above does not contain all the points of $A$ (the intersection condition is not satisfied)? For example consider $Z=\left\{x, a_{1}, a_{2}, a_{3}, a_{4}, y\right\}$ with the distance function depicted by the following diagram, where the dotted lines indicate distance 2 and the continuous lines indicate distance 1 :

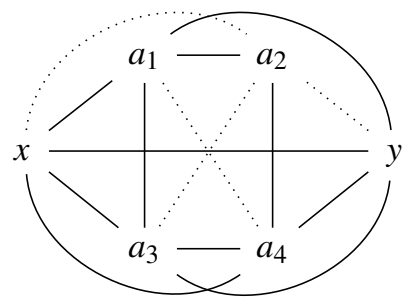

Let $X=\left\{x, a_{1}, a_{2}, a_{3}, a_{4}\right\}$ and $Y=\left\{a_{1}, a_{2}, a_{3}, a_{4}, y\right\}$. Choose $r=1$. In this case $\mathrm{VR}_{1}(Z)_{1} \backslash P$ consists of only one edge $\{x, y\}$ and $\operatorname{St}(\{x, y\}, A)=\Delta\left[\left\{a_{1}, a_{3}\right\}\right] \cup$ $\Delta\left[\left\{a_{3}, a_{4}\right\}\right]$. Thus condition 2 of Proposition 11.2 is not satisfied. However, since the complex $\operatorname{St}(\{x, y\}, A)$ is contractible, according to Corollary 8.7.1, the inclusion $\operatorname{VR}_{1}(X) \cup \operatorname{VR}_{1}(Y) \subset \operatorname{VR}_{1}(Z)$ is a weak equivalence.

Assumption 1 of Proposition 11.2 can be restated as: (connectivity condition) for every $\tau$ in $\operatorname{VR}_{r}(Z)_{1} \backslash P$, the complex $\operatorname{St}(\tau, A)$ is $n$-connected, and (independence condition) for all pairs of edges $\tau_{1}$ and $\tau_{2}$ in $\operatorname{VR}_{r}(Z)_{1} \backslash P$, there is an equality $\operatorname{St}\left(\tau_{1}, A\right)=\operatorname{St}\left(\tau_{2}, A\right)$. What if the independence condition is not satisfied? For example consider a distance space $Z=\left\{x_{1}, x_{2}, a_{1}, a_{2}, a_{3}, a_{4}, y\right\}$ with the distance function depicted by the following diagram, where the dotted lines indicate distance 2 and the continuous lines indicate distance 1 : 


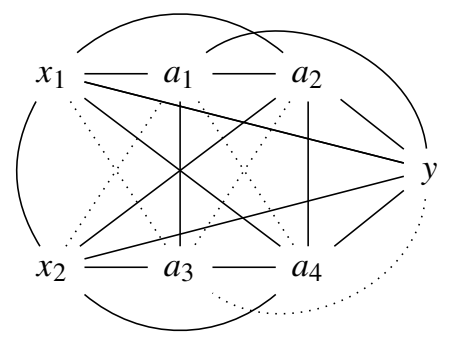

Let $X=\left\{x_{1}, x_{2}, a_{1}, a_{2}, a_{3}, a_{4}\right\}$ and $Y=\left\{a_{1}, a_{2}, a_{3}, a_{4}, y\right\}$. Choose $r=1$. In this case $\operatorname{VR}_{1}(Z)_{1} \backslash P$ consists of two edges $\left\{x_{1}, y\right\}$ and $\left\{x_{2}, y\right\}$. Note that $\operatorname{St}\left(\left\{x_{1}, y\right\}, A\right)=\Delta\left[\left\{a_{1}, a_{2}\right\}\right] \cup \Delta\left[\left\{a_{2}, a_{4}\right\}\right]$ and $\operatorname{St}\left(\left\{x_{2}, y\right\}, A\right)=\Delta\left[\left\{a_{2}, a_{4}\right\}\right]$. Thus the independence condition does not hold in this case. However, since the obstruction complexes $\operatorname{St}\left(\left\{x_{1}, y\right\}, A\right), \operatorname{St}\left(\left\{x_{2}, y\right\}, A\right)$ and $\operatorname{St}\left(\left\{x_{1}, x_{2}, y\right\}, A\right)=\operatorname{St}\left(\left\{x_{1}, y\right\}, A\right) \cap$ $\operatorname{St}\left(\left\{x_{2}, y\right\}, A\right)=\Delta\left[\left\{a_{2}, a_{4}\right\}\right]$ are contractible, the inclusion $\operatorname{VR}_{1}(X) \cup \operatorname{VR}_{1}(Y) \subset$ $\mathrm{VR}_{1}(Z)$ is a weak equivalence by Corollary 8.7.

Consider a relaxation of the intersection condition in assumption 2 of Proposition 11.2.

11.3 (Assumption I) Notation as in 11.1. Let $r$ be an element in $[0, \infty)$. There exists an element $v$ in $A$ satisfying the following property. For all $x$ in $X \backslash A$ and $y$ in $Y \backslash A$, if $d(x, y) \leq r$, then $d(x, v) \leq r$ and $d(y, v) \leq r$.

11.4 The assumption 11.3 is equivalent to non-emptiness of the following intersection, where $n \geq 0$ and the first equality is a consequence of Vietoris-Rips complexes being clique (see Proposition 10.1.3):

$$
\bigcap_{\sigma \in\left(\mathrm{sk}_{n+1} \mathrm{VR}_{r}(Z)\right) \backslash P} \operatorname{St}(\sigma, A)=\bigcap_{\sigma \in \mathrm{VR}_{r}(Z)_{1} \backslash P} \operatorname{St}(\sigma, A) \neq \emptyset
$$

According to Proposition 9.1, Assumption 11.3 implies connectedness of the homotopy fibers of $\mathrm{VR}_{r}(X) \cup \mathrm{VR}_{r}(Y) \subset \mathrm{VR}_{r}(Z)$. This assumption however does not imply $n$-connectedness of these homotopy fibers. For example consider $Z=\{x, a, b, y\}$ with the distance function depicted by the following diagram where the dotted line indicates distance 2 and the continuous lines indicate distance 1 :

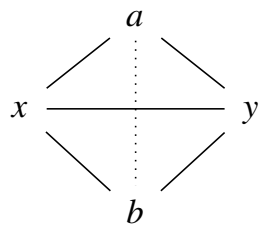

Let $X=\{x, a, b\}$ and $Y=\{a, b, y\}$. Then $\operatorname{VR}_{1}(Z)$ is contractible but $\operatorname{VR}_{1}(X) \cup$ $\operatorname{VR}_{1}(Y)$ has the homotopy type of a circle. Thus in this case the homotopy fiber of the inclusion $\operatorname{VR}_{1}(X) \cup \mathrm{VR}_{1}(Y) \subset \mathrm{VR}_{1}(Z)$ is not 1-connected. Note further that the complex $\operatorname{St}(\{x, y\}, A)$ consists of two vertices $a$ and $b$ with no edges. 
To assure $\operatorname{VR}_{r}(X) \cup \mathrm{VR}_{r}(Y) \hookrightarrow \mathrm{VR}_{r}(Z)$ is a weak equivalence assumption 11.3 is not enough and we need additional requirements. For example the following is an analogue of Corollary 10.4 .

Proposition 11.5 Notation as in 11.1. Assume 11.3. Let $v$ be an element in A given by this assumption. In addition assume that one of the following conditions is satisfied:

1. For every $x$ in $X \backslash A$ and $y$ in $Y \backslash A$ such that $d(x, y) \leq r$, if $w$ in A satisfies $d(w, x) \leq r$ and $d(w, y) \leq r$, then $d(v, w) \leq r$.

2. $\operatorname{diam}(A) \leq r$.

3. $A=\{v\}$.

Then the inclusion $V R_{r}(X) \cup V R_{r}(Y) \hookrightarrow V R_{r}(Z)$ is a weak equivalence.

Proof If Assumption 11.3 and condition 1 hold, then so does assumption 1 of Proposition 10.5. Furthermore condition 3 implies 2 and condition 2 implies 1 . Thus this proposition is a consequence of Proposition 10.5.1.

The intersection condition of assumption 2 in Proposition 11.2 requires a choice of a parameter $r$. The following is its universal version where no parameter is required:

11.6 (Assumption II) Notation as in 11.1. The set $A$ is non empty and for every $x$ in $X \backslash A, y$ in $Y \backslash A$, and $v$ in $A$, the following inequalities hold $d(x, y) \geq d(x, v)$ and $d(x, y) \geq d(y, v)$.

Assumption 11.6 has an intuitive interpretation in terms of angles when $Z$ is a subspace of the Euclidean space. In such a setting this condition means that every triangle $x v y$ with vertices $x$ in $X \backslash A, y$ in $Y \backslash A$ and $v$ in $A$, the angle at $v$ must be at least $60^{\circ}$. We therefore call this assumption the $60^{\circ}$ angle condition.

Proposition 11.7 Notation as in 11.1. Assume 11.6. Assume in addition that, for every $x$ in $X \backslash A$ and $y$ in $Y \backslash A$, the following inequality holds $d(x, y) \geq \operatorname{diam}(A)$. Then $V R_{r}(X) \cup V R_{r}(Y) \hookrightarrow V R_{r}(Z)$ is a weak equivalence for all $r$ in $[0, \infty)$.

Proof We already know that the proposition holds if $r \geq \operatorname{diam}(A)$ (see Proposition 11.5). Assume $r<\operatorname{diam}(A)$. We claim that in this case $\mathrm{VR}_{r}(Z)=P$ (see 8.2). If not, there are $x$ in $X \backslash A$ and $y$ in $Y \backslash A$ such that $d(x, y) \leq r$. The assumption would then lead to the following contradictory inequalities $r \geq d(x, y) \geq \operatorname{diam}(A)>r$. Thus in this case $\operatorname{VR}_{r}(Z)=P$ and the proposition follows from Proposition 8.3.

\section{Metric gluings}

A distance $d$ on $Z$ is called a pseudometric if it satisfies the triangle inequality: $d(x, z) \leq d(x, y)+d(y, z)$ for all $x, y, z$ in $Z$.

12.1 Notation as in 11.1. Assume that the distance $d$ on $Z$ is a pseudometric. Let $x$ be in $X \backslash A$ and $y$ be in $Y \backslash A$. For all $a$ in $A$, by the triangular inequality, $d(x, y) \leq$ $d(x, a)+d(a, y)$, and hence: 


$$
d(x, y) \leq \inf \{d(x, a)+d(a, y) \mid a \in A\}
$$

The pseudometric space $(Z, d)$ is called metric gluing if the above inequality is an equality for all $x$ in $X \backslash A$ and $y$ in $Y \backslash A$.

If $A$ is finite, then the pseudometric $(Z, d)$ is a metric gluing if and only if, for every $x$ in $X \backslash A$ and $y$ in $Y \backslash A$, there is $a$ in $A$ such that $d(x, y)=d(x, a)+d(a, y)$.

If $d_{X}$ is a pseudometric on $X$ and $d_{Y}$ is a pseudometric on $Y$ such that $d_{X}(a, b)=$ $d_{Y}(a, b)$ for all $a$ and $b$ in $A$, then the following function defines a pseudometric on $Z$ which is a metric gluing:

$$
d_{Z}\left(z, z^{\prime}\right)= \begin{cases}d_{X}\left(z, z^{\prime}\right) & \text { if } z, z^{\prime} \in X \\ d_{Y}\left(z, z^{\prime}\right) & \text { if } z, z^{\prime} \in Y \\ \inf \left\{d(z, a)+d\left(z^{\prime}, a\right) \mid a \in A\right\} & \text { if } z \in X \backslash A \text { and } z^{\prime} \in Y \backslash A\end{cases}
$$

If $(Z, d)$ is a metric gluing and $A$ is finite, then, for any edge $\sigma=\{x, y\}$ in $\operatorname{VR}_{r}(Z) \backslash P$, there is $a$ in $A$ such that $r \geq d(x, y)=d(x, a)+d(a, y)$. Thus in this case the obstruction complex $\operatorname{St}(\tau, A)$ is non-empty as it contains the vertex $a$. To assure contractibility of $\operatorname{St}(\tau, A)$ we need additional assumptions, for example:

12.2 (Simplex assumption) Notation as in 11.1 and 8.2. Let $r$ be in $[0, \infty)$. For any vertex $v$ in an edge $\sigma$ in $\mathrm{VR}_{r}(Z) \backslash P$, if a and $b$ are elements in $A$ such that $d(a, v) \leq r$ and $d(v, b) \leq r$, then $d(a, b) \leq r$.

The simplex assumption can be reformulated as follows: for any vertex $v$ in a simplex $\sigma$ in $\operatorname{VR}_{r}(Z) \backslash P$, the complex $\operatorname{St}(v, A)$ is a standard simplex (see 4.1). Since the intersection of standard simplices is again a standard simplex, under Assumption 12.2, an obstruction complex $\operatorname{St}(\sigma, A)$, for an arbitrary simplex $\sigma$ in $\operatorname{VR}_{r}(Z) \backslash P$, is contractible if and only if it is non empty. This, together with the discussion at the end of 12.1 and Corollary 10.2 gives:

Proposition 12.3 Notation as in 11.1 and 8.2. Letr be in $[0, \infty)$. Assume A is finite and $(Z, d)$ is a metric gluing that satisfies the simplex assumption 12.2. Then, for any edge $\sigma$ in $V R_{r}(Z) \backslash P$, the obstruction complex $\operatorname{St}(\sigma, A)$ is contractible. The homotopy fibers of $V R_{r}(X) \cup V R_{r}(Y) \hookrightarrow V R_{r}(Z)$ are connected and this map induces an isomorphism on $\pi_{0}$ and a surjection on $\pi_{1}$.

The assumptions of Proposition 12.3 are not enough to guarantee the non-emptiness of the obstruction complexes for simplices in $\operatorname{VR}_{r}(Z) \backslash P$ of dimension 2 and higher. For example consider $Z=\left\{x_{1}, x_{2}, a_{1}, a_{1}, y\right\}$ with the distance function depicted by the following diagram, where the dotted lines indicate distance 4 , the dashed lines indicate distance 3 , the squiggly lines indicate distance 2 and the continuous lines indicate distance 1 : 


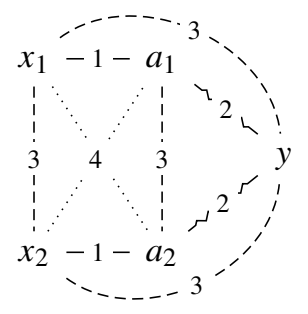

Let $r=3, X=\left\{x_{1}, x_{2}, a_{1}, a_{2}\right\}, Y=\left\{y, a_{1}, a_{2}\right\}$, and $A=\left\{a_{1}, a_{2}\right\}$. Then $Z$ is a metric gluing. Note that $\operatorname{St}(y, A)=\Delta\left[\left\{a_{1}, a_{2}\right\}\right], \operatorname{St}\left(x_{1}, A\right)=\operatorname{St}\left(\left\{x_{1}, y\right\}, A\right)=\Delta\left[\left\{a_{1}\right\}\right]$, $\operatorname{St}\left(x_{2}, A\right)=\operatorname{St}\left(\left\{x_{2}, y\right\}, A\right)=\Delta\left[\left\{a_{2}\right\}\right]$, and $\operatorname{St}\left(\left\{x_{1}, x_{2}, y\right\}, A\right)$ is empty. Furthermore $A$ and $Y$ have diameter not exceeding 3. Consequently, $\mathrm{VR}_{3}(Y)$ and $\mathrm{VR}_{3}(A)$ are contractible. The complex $\mathrm{VR}_{3}(X)$ has the homotopy type of the circle $S^{1}$ and so does $\mathrm{VR}_{3}(X) \cup \mathrm{VR}_{3}(Y)$. The entire complex $\mathrm{VR}_{3}(Z)$ is however contractible. The inclusion $\mathrm{VR}_{3}(X) \cup \mathrm{VR}_{3}(Y) \subset \mathrm{VR}_{3}(Z)$ induces therefore a surjection on $\pi_{1}$ but not an isomorphism. This example should be compared with Proposition 11.5 under condition 2 .

To assure isomorphism on $\pi_{1}$, the simplex assumption 12.2 should be strengthened.

12.4 (Strong simplex assumption) Notation as in 11.1 and 8.2. Let $r$ be in $[0, \infty)$. For any vertex $v$ in an edge $\sigma$ in $\operatorname{VR}_{r}(Z) \backslash P$, if $a$ and $b$ are elements in $A$ such that $d(a, v) \leq r$ and $d(v, b) \leq r$, then $2 d(a, b) \leq d(a, v)+d(v, b)$.

Note that the strong simplex assumption 12.4 implies the simplex assumption 12.2.

Theorem 12.5 Notation as in 11.1 and 8.2. Let $r$ be in $[0, \infty)$. Assume $A$ is finite and $(Z, d)$ is a metric gluing that satisfies the strong simplex assumption 12.4. Then, for any simplex $\sigma$ in $V R_{r}(Z) \backslash P$ such that either $|\sigma \cap X|=1$ or $|\sigma \cap Y|=1$, the obstruction complex $\operatorname{St}(\sigma, A)$ is contractible. The homotopy fibers of the inclusion $V R_{r}(X) \cup$ $V R_{r}(Y) \hookrightarrow V R_{r}(Z)$ are simply connected and this map induces an isomorphism on $\pi_{0}$ and $\pi_{1}$ and a surjection on $\pi_{2}$.

Proof Since the strong simplex assumption 12.4 is satisfied, then so is the simplex assumption 12.2 and consequently any obstruction complex $\operatorname{St}(\sigma, A)$ is a simplex. Thus $\operatorname{St}(\sigma, A)$ is contractible if and only if it is non empty.

We are going to show by induction on the dimension of a simplex a more general statement:

Under the assumption of Theorem 12.5, for every simplex $\sigma$ in $\mathrm{VR}_{r}(Z) \backslash P$ for which $\sigma \cap X=\left\{x_{1}, \ldots, x_{n}\right\}$ and $\sigma \cap Y=\{y\}$, if $\left(a_{1}, \ldots, a_{n}\right)$ is a sequence in $A$ such that $d\left(x_{i}, y\right)=d\left(x_{i}, a_{i}\right)+d\left(a_{i}, y\right)$ for every $i$, then there is an integer $1 \leq l \leq n$ for which $a_{l}$ is in $\operatorname{St}(\sigma, A)\left(d\left(x_{i}, a_{l}\right) \leq r\right.$ for all $\left.i\right)$.

If $\sigma=\{x, y\}$ is such an edge, then the statement is clear.

Let $n>1$ and assume that the statement is true for all relevant simplices of dimension smaller than $n$. Let $\sigma$ be in $\operatorname{VR}_{r}(Z) \backslash P$ be such that $\sigma \cap X=\left\{x_{1}, \ldots, x_{n}\right\}$ and $\sigma \cap Y=\{y\}$. Choose a sequence $\left(a_{1}, \ldots, a_{n}\right)$ in $A$ such that $d\left(x_{i}, y\right)=d\left(x_{i}, a_{i}\right)+$ $d\left(y, a_{i}\right)$ for every $i$. 
By the inductive assumption, for every $j=1, \ldots, n$, the statement is true for $\tau_{j}=\sigma_{j} \backslash\left\{x_{j}\right\}$ and the sequence $\left(a_{1}, \ldots, \widehat{a_{j}}, \ldots, a_{n}\right)$ obtained from $\left(a_{1}, \ldots, a_{n}\right)$ by removing its $j$-th element. Thus for every $j=1, \ldots, n$, there is $a_{s(j)}$ such that $s(j) \neq j$ and $d\left(x_{i}, a_{s(j)}\right) \leq r$ for all $i \neq j$. If, for some $j, d\left(x_{j}, a_{s(j)}\right) \leq r$, then $a_{s(j)}$ would be a vertex in $\operatorname{St}(\sigma, A)$, proving the statement. Assume $d\left(x_{j}, a_{s(j)}\right)>r$ for all $j$. If $j \neq j^{\prime}$, then $d\left(x_{j}, a_{s\left(j^{\prime}\right)}\right) \leq r$ and $d\left(x_{j}, a_{s(j)}\right)>r$, and hence $a_{s(j)} \neq a_{s\left(j^{\prime}\right)}$. It follows that $s$ is a permutation of the set $\{1, \ldots, n\}$. This leads to a contradictory inequality:

$$
\begin{aligned}
n r & <\sum_{i=1}^{n} d\left(x_{i}, a_{s(i)}\right) \leq \sum_{i=1}^{n}\left(d\left(x_{i}, a_{i}\right)+d\left(a_{i}, a_{s(i)}\right)\right) \\
& \leq \sum_{i=1}^{n}\left(d\left(x_{i}, a_{i}\right)+\frac{1}{2} d\left(a_{i}, y\right)+\frac{1}{2} d\left(y, a_{s(i)}\right)\right) \\
& \leq \sum_{i=1}^{n}\left(d\left(x_{i}, a_{i}\right)+d\left(y, a_{i}\right)\right)=\sum_{i=1}^{n} d\left(x_{i}, y\right) \leq n r
\end{aligned}
$$

In the above chain of inequalities the second one is obtained by applying triangular inequality, the third one is the strong simplex assumption and the fourth one is a consequence of the fact that $s$ is a permutation. Note that, for any simplex $\sigma$ in $\operatorname{sk}_{2} \operatorname{VR}(Z) \backslash P$, either $|\sigma \cap X|=1$ or $|\sigma \cap Y|=1$. Thus, for any such simplex, the obstruction complex $\operatorname{St}(\sigma, A)$ is contractible. We can then use Proposition 8.8 to conclude that the homotopy fibers of the inclusion $\operatorname{VR}_{r}(X) \cup \mathrm{VR}_{r}(Y) \hookrightarrow \mathrm{VR}_{r}(Z)$ are simply connected.

The conclusion of Theorem 12.5 is sharp. Its assumptions are not enough to assure that the homotopy fibers of the map $\operatorname{VR}_{r}(X) \cup \mathrm{VR}_{r}(Y) \hookrightarrow \mathrm{VR}_{r}(Z)$ are 2-connected. We finish this section with an example illustrating this fact.

12.6 Let $Z=\left\{x_{1}, x_{2}, a_{11}, a_{12}, a_{21}, a_{22}, y_{1}, y_{2}\right\}, X=\left\{x_{1}, x_{2}, a_{11}, a_{12}, a_{21}, a_{22}\right\}$ and $Y=\left\{y_{1}, y_{2}, a_{11}, a_{12}, a_{21}, a_{22}\right\}$. Consider the distance function $d$ on $Z$ described by the following table:

\begin{tabular}{c|c|c|c|c|c|c|c} 
& $x_{2}$ & $a_{11}$ & $a_{12}$ & $a_{21}$ & $a_{22}$ & $y_{1}$ & $y_{2}$ \\
\hline$x_{1}$ & 6 & 3 & 5 & 7 & 9 & 8 & 8 \\
\hline$x_{2}$ & 0 & 9 & 7 & 5 & 3 & 8 & 8 \\
\hline$a_{11}$ & & 0 & 4 & 4 & 6 & 5 & 7 \\
\hline$a_{12}$ & & & 0 & 6 & 4 & 9 & 3 \\
\hline$a_{21}$ & & & & 0 & 4 & 3 & 9 \\
\hline$a_{22}$ & & & & & 0 & 7 & 5 \\
\hline$y_{1}$ & & & & & & 0 & 6
\end{tabular}

The distance $d$ satisfies the triangular inequality and hence $(Z, d)$ is a metric space. Furthermore $d\left(x_{i}, y_{j}\right)=d\left(x_{i}, a_{i j}\right)+d\left(a_{i j}, y_{j}\right)$ for any $i$ and $j$. Thus $(Z, d)$ is a metric gluing of $X$ and $Y$. The metric space $(Z, d)$ can be represented by the following 
diagram, where the continuous lines or no line indicate distance 8 or smaller and the dotted lines indicate distance 9:

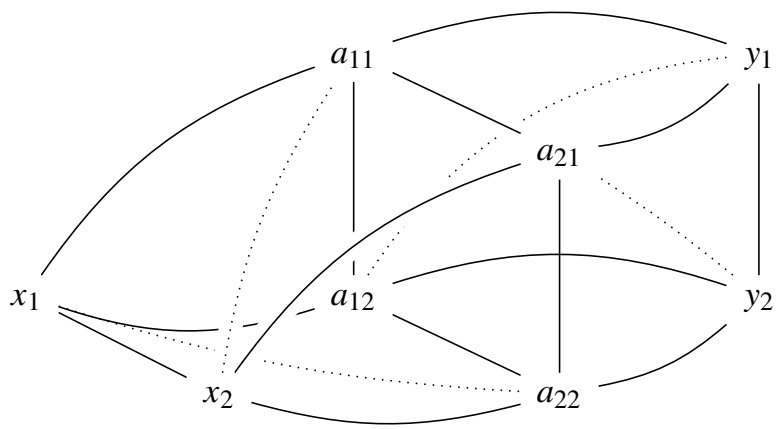

By direct calculation one checks that, for $r=8$ and $Z=X \cup Y$, the metric space $(Z, d)$ satisfies the strong simplex assumption 12.4. However, the complex $\operatorname{VR}_{8}(X) \cup \operatorname{VR}_{8}(Y)$ is contractible and $\mathrm{VR}_{8}(Z)$ is weakly equivaent to $S^{3}$ (3-dimensional sphere).The homotopy fiber of $\mathrm{VR}_{8}(X) \cup \mathrm{VR}_{8}(Y) \subset \mathrm{VR}_{8}(Z)$ is therefore weakly equivalent to the loops space $\Omega S^{3}$ and hence is not 2 connected.

Here are steps that one might use to see that $\mathrm{VR}_{8}(Z)$ is weakly equivalent to $S^{3}$. Consider the simplex $\left\{x_{1}, x_{2}\right\}$ in $\mathrm{VR}_{8}(Z)$. According to Corollary 7.2 , there is a homotopy cofiber sequence:

$$
\Sigma \mathrm{St}\left(\left\{x_{1}, x_{2}\right\}, \mathrm{VR}_{8}\left(Z \backslash\left\{x_{1}, x_{2}\right\}\right) \rightarrow \mathrm{VR}_{8}\left(Z \backslash\left\{x_{1}\right\}\right) \cup \mathrm{VR}_{8}\left(Z \backslash\left\{x_{2}\right\}\right) \rightarrow \mathrm{VR}_{8}(Z)\right.
$$

The complexes in this sequence have the following homotopy types:

- $\operatorname{St}\left(\left\{x_{1}, x_{2}\right\}, \operatorname{VR}_{8}\left(Z \backslash\left\{x_{1}, x_{2}\right\}\right)\right.$ is weakly equivalent to the circle $S^{1}$;

- $\mathrm{VR}_{8}\left(Z \backslash\left\{x_{1}\right\}\right), \mathrm{VR}_{8}\left(Z \backslash\left\{x_{2}\right\}\right), \mathrm{VR}_{8}\left(Z \backslash\left\{x_{1}, x_{2}\right\}\right)$ are contractible;

- the above implies that $\mathrm{VR}_{8}\left(Z \backslash\left\{x_{1}\right\}\right) \cup \mathrm{VR}_{8}\left(Z \backslash\left\{x_{2}\right\}\right)$ is also contractible;

- we can then use the cofiber sequence above to conclude $\operatorname{VR}_{8}(Z)$ is weakly equivalent to $\Sigma^{2} S^{1} \simeq S^{3}$ as claimed. It is also possible to see this by noticing that $\mathrm{VR}_{8}(Z)$ is homeomorphic to the boundary of the cross-polytope on 8 vertices, since all edges except the antipodal ones are present.

\section{Vietoris-Rips of 9 points on a circle}

In Adamaszek (2013), Adamaszek and Adams (2017), Adamaszek et al. (2016) a lot of techniques were introduced aiming at describing homotopy types of certain VietorisRips complexes, particularly for metric graphs built from points on a circle. In this section we showcase how our techniques can be used to describe the homotopy type of one of such examples. Consider a metric space given by 9 points, $Z=\left\{z_{i}\right\}_{i}$ with the following distances between them: 
Fig. 3 Only some of the edges of the simplicial complex are represented. In particular the edges connecting points $a_{1}, a_{2}$ and $a_{3}$ in $A$, having distance 3 between each other, are represented as straight lines

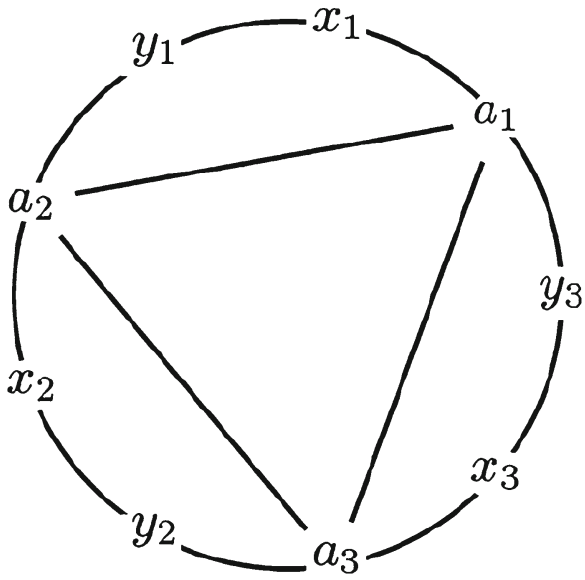

\begin{tabular}{c|c|c|c|c|c|c|c|c} 
& $x_{1}$ & $y_{1}$ & $a_{2}$ & $x_{2}$ & $y_{2}$ & $a_{3}$ & $x_{3}$ & $y_{3}$ \\
\hline$a_{1}$ & 1 & 2 & 3 & 4 & 4 & 3 & 2 & 1 \\
\hline$x_{1}$ & 0 & 1 & 2 & 3 & 4 & 4 & 3 & 2 \\
\hline$y_{1}$ & & 0 & 1 & 2 & 3 & 4 & 4 & 3 \\
\hline$a_{2}$ & & & 0 & 1 & 2 & 3 & 4 & 4 \\
\hline$x_{2}$ & & & & 0 & 1 & 2 & 3 & 4 \\
\hline$y_{2}$ & & & & & 0 & 1 & 2 & 3 \\
\hline$a_{3}$ & & & & & & 0 & 1 & 2 \\
\hline$x_{3}$ & & & & & & & 0 & 1
\end{tabular}

This metric space can be visualised as a metric graph consisting of 9 points on a circle, where distances between adjacent points are set to be 1 .

We are going to illustrate how to use our techniques to prove that $\mathrm{VR}_{3}(Z)$ is weakly equivalent to the wedge $S^{2} \vee S^{2}$ of two 2-dimensional spheres, a result already present in the mentioned work of Adamaszek et al. Set $X:=\left\{x_{1}, x_{2}, x_{3}, a_{1}, a_{2}, a_{3}\right\}$ and $Y:=\left\{y_{1}, y_{2}, y_{3}, a_{1}, a_{2}, a_{3}\right\}$. Note that $X \cup Y=Z$ and $A:=X \cap Y=\left\{a_{1}, a_{2}, a_{3}\right\}$.

Note that $\operatorname{VR}_{3}(X \cap Y)$ is contractible, and thus $\operatorname{VR}_{3}(X) \cup \mathrm{VR}_{3}(Y)$ is homotopy equivalent to the wedge $\mathrm{VR}_{3}(X) \vee \mathrm{VR}_{3}(Y)$. Furthermore we claim that all the obstruction complexes $\operatorname{St}(\sigma, A)$ are contractible for all simplices $\sigma$ in $\mathrm{VR}_{3}(Z)$ such that $\sigma \cap X \neq \emptyset, \sigma \cap Y \neq \emptyset$, and $\sigma \cap X \cap Y=\emptyset$. For example if $\sigma=\left\{x_{1}, y_{1}\right\}$ then $\operatorname{St}(\sigma, A)=\Delta\left[a_{1}, a_{2}\right]$ which is contractible. According to Corollary 8.7.1, the inclusion $V R_{3}(X) \cup V R_{3}(Y) \subset V R_{3}(Z)$ is therefore a weak equivalence and consequently $V R_{3}(Z)$ has the homotopy type of the wedge $\operatorname{VR}_{3}(X) \vee \mathrm{VR}_{3}(Y)$. The metric spaces $X$ and $Y$ are isometric, and hence the corresponding Vietoris-Rips complexes are isomorphic. It remains to show that $V R_{3}(X)$ has the homotopy type of $S^{2}$. Consider $X^{\prime}=\left\{a_{1}, x_{2}\right\}$ and $X^{\prime \prime}=\left\{x_{1}, a_{2}, x_{3}, y_{3}\right\}$. Note that $X=X^{\prime} \bigsqcup X^{\prime \prime}, \operatorname{VR}_{3}\left(X^{\prime}\right)$ has the homotopy type of $S^{0}$ and $\mathrm{VR}_{3}\left(X^{\prime \prime}\right)$ has the homotopy type of $S^{1}$. Finally note that, for all simplices $\sigma$ in $\operatorname{VR}_{3}\left(X^{\prime}\right)$ and $\mu$ in $\operatorname{VR}_{3}\left(X^{\prime \prime}\right)$, the union $\sigma \cup \mu$ is a simplex in 
$V R_{3}(X)$. This implies that $V R_{3}(X)$ is the join of $\operatorname{VR}_{3}\left(X^{\prime}\right)$ and $\mathrm{VR}_{3}\left(X^{\prime \prime}\right)$ (see Paragraph 4.11) and hence it is weakly equivalent to $\Sigma\left(S^{0} \wedge S^{1}\right) \simeq S^{2}$. As in the previous example, it is also possible to see this by noticing that $\operatorname{VR}_{3}(X)$ is homeomorphic to the boundary of the cross-polytope on 6 vertices, since all edges except the antipodal ones are present.

Acknowledgements A. Jin and F. Tombari were supported by the Wallenberg AI, Autonomous System and Software Program (WASP) funded by Knut and Alice Wallenberg Foundation. W. Chachólski was partially supported by VR and WASP. M. Scolamiero was partially supported by Brummer \& Partners MathDataLab and WASP.

Funding Open access funding provided by Royal Institute of Technology.

\section{Declarations}

Conflict of interest On behalf of all authors, the corresponding author states that there is no conflict of interest.

Open Access This article is licensed under a Creative Commons Attribution 4.0 International License, which permits use, sharing, adaptation, distribution and reproduction in any medium or format, as long as you give appropriate credit to the original author(s) and the source, provide a link to the Creative Commons licence, and indicate if changes were made. The images or other third party material in this article are included in the article's Creative Commons licence, unless indicated otherwise in a credit line to the material. If material is not included in the article's Creative Commons licence and your intended use is not permitted by statutory regulation or exceeds the permitted use, you will need to obtain permission directly from the copyright holder. To view a copy of this licence, visit http://creativecommons.org/licenses/by/4.0/.

\section{References}

Aanjaneya, M., Chazal, F., Chen, D., Glisse, M., Guibas, L., Morozov, D.: Metric graph reconstruction from noisy data. In: Computational geometry (SCG'11), pp. 37-46. ACM, New York (2011). https://doi. org/10.1145/1998196.1998203

Adamaszek, M.: Clique complexes and graph powers. Israel J. Math. 196(1), 295-319 (2013)

Adamaszek, M.: Small flag complexes with torsion. Canad. Math. Bull. 57(2), 225-230 (2014). https://doi. org/10.4153/CMB-2013-032-9

Adamaszek, M., Adams, H.: The Vietoris-Rips complexes of a circle. Pacific J. Math. 290(1), 1-40 (2017)

Adamaszek, M., Adams, H., Frick, F., Peterson, C., Previte-Johnson, C.: Nerve complexes of circular arcs. Discrete Comput. Geom. 56(2), 251-273 (2016)

Adamaszek, M., Adams, H., Gasparovic, E., Gommel, M., Purvine, E., Sazdanovic, R., Wang, B., Wang, Y., Ziegelmeier, L.: Vietoris-Rips and Čech complexes of metric gluings. In: 34th International Symposium on Computational Geometry, LIPIcs. Leibniz Int. Proc. Inform., vol. 99, pp. Art. No. 3, 15 (2018)

Adamaszek, M., Adams, H., Gasparovic, E., Gommel, M., Purvine, E., Sazdanovic, R., Wang, B., Wang, Y., Ziegelmeier, L.: On homotopy types of Vietoris-Rips complexes of metric gluings. J. Appl. Comput. Topol. 4(3), 425-454 (2020). https://doi.org/10.1007/s41468-020-00054-y

Bubenik, P.: Statistical topological data analysis using persistence landscapes. J. Mach. Learn. Res. 16, 77-102 (2015)

Cagliari, F., Ferri, M., Pozzi, P.: Size functions from a categorical viewpoint. Acta Appl. Math. 67(3), 225-235 (2001)

Carlsson, G.: Topology and data. Bull. Amer. Math. Soc. (N.S.) 46(2), 255-308 (2009)

Carlsson, G., Zomorodian, A., Collins, A., Guibas, L.: Persistence barcodes for shapes. Int. J. Shape Model. 11(2), 149-187 (2005) 
Chachólski, W.: Closed classes. In: Algebraic topology: new trends in localization and periodicity (Sant Feliu de Guíxols, 1994), Progr. Math., vol. 136, pp. 95-118. Birkhäuser, Basel (1996)

Chachólski, W., Riihimäki, H.: Metrics and Stabilization in One Parameter Persistence. SIAM J. Appl. Algebra Geom. 4(1), 69-98 (2020)

Curtis, E.: Simplicial homotopy theory. Aarhus Universitet, Aarhus, Matematisk Institut (1967)

Delfinado, C.J.A., Edelsbrunner, H.: An incremental algorithm for betti numbers of simplicial complexes on the 3-sphere. Comput. Aided Geom. Design 12, 771-784 (1995)

Dror Farjoun, E.: Cellular inequalities. In: The Čech centennial (Boston, MA, 1993), Contemp. Math., vol. 181, pp. 159-181. Amer. Math. Soc., Providence, RI (1995)

Edelsbrunner, H., Harer, J.: Persistent homology—a survey. In: Surveys on discrete and computational geometry, Contemp. Math., vol. 453, pp. 257-282 (2008)

Farjoun, E.D.: Cellular spaces, null spaces and homotopy localization. Lecture Notes in Mathematics, vol. 1622. Springer-Verlag, Berlin (1996)

Ferri, M.: Size functions: a new topological approach to shape analysis. In: Geometry and topology of submanifolds. VII (Leuven, 1994/Brussels, 1994), pp. 299-304. World Sci. Publ, River Edge, NJ (1995)

Frosini, P., Landi, C.: Size theory as a topological tool for computer vision. Pattern Recognit. Image Anal. 9, 596-603 (1999)

Goerss, P.G., Jardine, J.F.: Simplicial homotopy theory, Progress in Mathematics, vol. 174. Birkhäuser Verlag, Basel (1999)

Hausmann, J.C.: On the Vietoris-Rips complexes and a cohomology theory for metric spaces. In: Prospects in topology (Princeton, NJ, 1994), Ann. of Math. Stud., vol. 138, pp. 175-188. Princeton Univ. Press, Princeton, NJ (1995)

Latch, D.M., Thomason, R.W., Wilson, W.S.: Simplicial sets from categories. Math. Z. 164(3), 195-214 (1979)

Maunder, C.: Algebraic Topology. Mineola, N.Y. : Dover Publications. Constable and Co, London (1996)

Munson, B., Volić, I.: Cubical Homotopy Theory. Cambridge University Press, (2015)

nlab: nerve. https://ncatlab.org/nlab/show/nerve

Scolamiero, M., Chachólski, W., Lundman, A., Ramanujam, R., Öberg, S.: Multidimensional persistence and noise. Found. Comput. Math. 17(6), 1367-1406 (2017). https://doi.org/10.1007/s10208-0169323-y

Taubes, C.H.: Metrics, connections and gluing theorems, CBMS Regional Conference Series in Mathematics, vol. 89. Published for the Conference Board of the Mathematical Sciences, Washington, DC; by the American Mathematical Society, Providence, RI (1996)

Publisher's Note Springer Nature remains neutral with regard to jurisdictional claims in published maps and institutional affiliations. 